\title{
Research Paper \\ The Mediational Roles of Law of Contagion and Threat Estimations in the Relation Between Disgust and Contamination-based OCD Symptoms
}

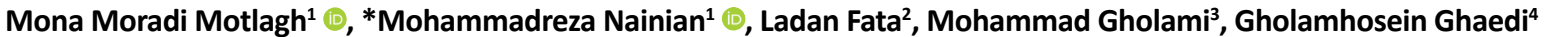

1. Department of Clinical Psychology, Faculty of Humanities, Shahed University, Tehran, Iran.

2. Department of Medical Education, Faculty of Medicine, Iran University of Medical Sciences, Tehran, Iran.

3. Department of Biological Statistics, Faculty of Medicine, Trbiat Modares University, Tehran, Iran.

4. Department of Psychiatry, Faculty of Medicine, Shahed University, Tehran, Iran.

\begin{tabular}{|c|c|}
\hline $\begin{array}{l}\text { Use vour device toscan } \\
\text { and read the article online }\end{array}$ & oradi Motlagh M, Nainian M, Fata L, Gholami M, Ghaedi Gh. [The Mediational Roles of Law of Contagion and \\
\hline 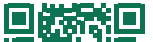 & Threat Estimations in the Relation Between Disgust and Contamination-based OCD Symptoms (Persian)]. Iranian Journal of \\
\hline 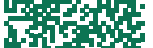 & Psychiatry and Clinical Psychology. 2020; 26(2):200-215. http://dx.doi.org/10.32598/ijpcp.26.2.3114.1 \\
\hline 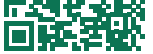 & doij http://dx.doi.org/10.32598/ijpcp.26.2.3114.1 \\
\hline
\end{tabular}

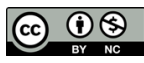

Received: 04 Jun 2019

Accepted: $12 \operatorname{Jan} 2020$

Available Online: $01 \mathrm{Jul} 2020$

Key words:

Disgust, Obsessive-

Compulsive Disorder

(OCD), Magic, Cogni-

tion, Models

\begin{abstract}
A B S T RACT
Objectives The law of contagion is one of the sympathetic of magic principles and is a cognitive distortion related to disgust. To explain how disgust can lead to contamination Obsessive-Compulsive Disorder (OCD) symptoms, this study assumes that the emotion of disgust can activate the law of contagion, which in turn may motivate threat estimations that finally results in OCD symptoms.

Methods This study has a correlational research design. Participants were 495 students (59\% women) from Olum Tahghighat University that recruited through convenience sampling. All the participants completed all the questionnaires in the same order: Threat estimation scenarios, the Vancouver Obsessional Compulsive Inventory Contamination Scale (VOCl-C), negative-spiritual contagion subscale from Contagion Sensitivity Scale (CSS), and core disgust subscale from Disgust Scale (DS). This model was examined through Structural Equation Modeling (SEM).

Results The finding revealed that the proposed model had a good fit based on reported indices: $\chi^{2}$, CMIN/DF, GFI, CFI, AGFI, RMSEA.

Conclusion There are mediational roles for the law of contagion and threat estimations in the relation between disgust and OCD symptoms. The proposed psychopathological model can help to promote the disgust theory in OCD and may have implications for cognitive behavioral therapy.
\end{abstract}

\section{Extended Abstract}

\section{Introduction}

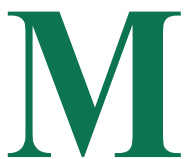

uch research has revealed that disgust is closely related to psychopathology [1]. Among psychiatry disorders, disgust has an important role in the genesis and maintenance of contamination-based OCD [2-6]. The relation between disgust and contamination OCD rises this question that how and with which mechanism, disgust can result in contamination OCD symptoms. According to Cisler et al [11]. Although disgust can explain the avoidance of direct contaminants in people with OCD, it is not enough for explaining the avoidance of indirect contaminants in OCD, and the role of cognitive variables should be implicated.

The most related cognitive distortions with disgust is the "sympathetic magic laws" [10], known more as the "law of contagion" [12-16]. This law states that people intend to behave as if a brief contact can lead to the permanent and

* Corresponding Author:

Mohammadreza Nainian, PhD.

Address: Department of Clinical Psychology, Faculty of Humanities, Shahed University, Tehran, Iran.

Tel: +98 (912) 7702588

E-mail: mrnainian@yahoo.com 
full transmission of characteristics or the "essence" from one object to the other one [12]. Woody and Tolin [17] suggested that this law is more elevated in OCD washers and analog samples. The exaggerated perception of magical contagion in OCD people, in turn, causes overestimations of threats including general danger, becoming contaminated, and getting ill. Verwoerd et al. [20] suggested that people with higher fear of contamination overestimated the likelihood of becoming ill more than the normal control group even in conditions with no objective threats but with disgust response.

Thus, in this study, the proposed structural model examined through SEM procedure, displayed that the activation of disgust can lead to the motivation of the law of contagion and that in turn causes motivation of threat overestimations, which finally result in contamination OCD symptoms.

\section{Methods}

This is a correlational research design. Participants were 495 (59\% women) students from Olum Tahghighat University which recruited through convenience sampling. The age range was 18 to 35 years old (Means \pm SD: 20.45 \pm 2.52 ). Most of them ranged from 18 to 20 (64\%).

\section{Procedure}

Students who were not busy in the time between classes were asked to participate in the research if they wished and completed all the questionnaires in the same order including threat estimations' scenarios, The Vancouver Obsessional Compulsive Inventory contamination subscale (VOCI-C), Negative Spiritual Contagion subscale from Contagion Sensitivity Scale (CSS), and Core Disgust subscale from Disgust Scale (DS-R).

\section{Analytic strategies}

The Means \pm SD and Pearson correlations as descriptive indicators were calculated. The structural model includes the relation between latent core disgust variable (its indicators were the subscale items) with latent fear of contamination variable (its indicators were obsessions and washing rituals) which was mediated by latent negative spiritual contagion variable (its indicators were the subscale items) and latent threat estimations variable (its indicators were the probability of danger, probability of becoming contaminated, and the probability of becoming ill). The model fit was examined through Structural Equation Modeling (SEM) procedures by the software of AMOS v. 18 while the following indices were reported: $\chi^{2}, \mathrm{CMIN} / \mathrm{DF}, \mathrm{GFI}, \mathrm{CFI}, \mathrm{AGFI}, \mathrm{RMSEA}$.

\section{Results}

In Table 1 the Means \pm SD and Pearson correlations were displayed for the variables in this study. The structural model was also shown in Figure 1 and the fit indices were as follows: $\chi^{2}=487.2, \mathrm{CMIN} / \mathrm{DF}=1.98, \mathrm{GFI}=0.925$, $\mathrm{AGFI}=0.91$, $\mathrm{CFI}=0.934$, and RMSEA $=0.045$. As CMIN/DF should be below 3, CFI, GFI and AGFI should be more than 0.90 and RMSEA should be below 0.06 [28, 29]. The model has a good fit especially based on CMIN/DF which is below 2 and RMSEA which is below 0.05 .

\section{Discussion}

The results revealed that the proposed structural model (Figure 1) has a good fit from which can be concluded that the magical law of contagion and threat estimations mediate the relation between core disgust and fear of contamination.

As mentioned before, to explain people's fear of indirect contaminants in OCD, cognitive variables should be considered $[11,30]$. Tolin et al. [15] found that OCD people's

Table 1. Means $\pm S D$ and Pearson correlations of the variables

\begin{tabular}{|c|c|c|c|c|c|c|c|}
\hline Variables & 1 & 2 & 3 & 4 & 5 & 6 & Means $\pm S D$ \\
\hline Danger estimation & 1 & 0.72 & 0.70 & 0.28 & 0.34 & 0.50 & $17.67 \pm 14.33$ \\
\hline Contamination estimation & & 1 & 0.86 & 0.30 & 0.40 & 0.53 & $23.22 \pm 14.95$ \\
\hline Illness estimation & & & 1 & 0.30 & 0.39 & 0.47 & $18.35 \pm 13.74$ \\
\hline Spiritual contagion & & & & 1 & 0.45 & 0.41 & $3.9 \pm 1.03$ \\
\hline Core disgust & & & & & 1 & 0.44 & $2.40 \pm 0.73$ \\
\hline OCD symptoms & & & & & & 1 & $1.67 \pm 0.72$ \\
\hline
\end{tabular}




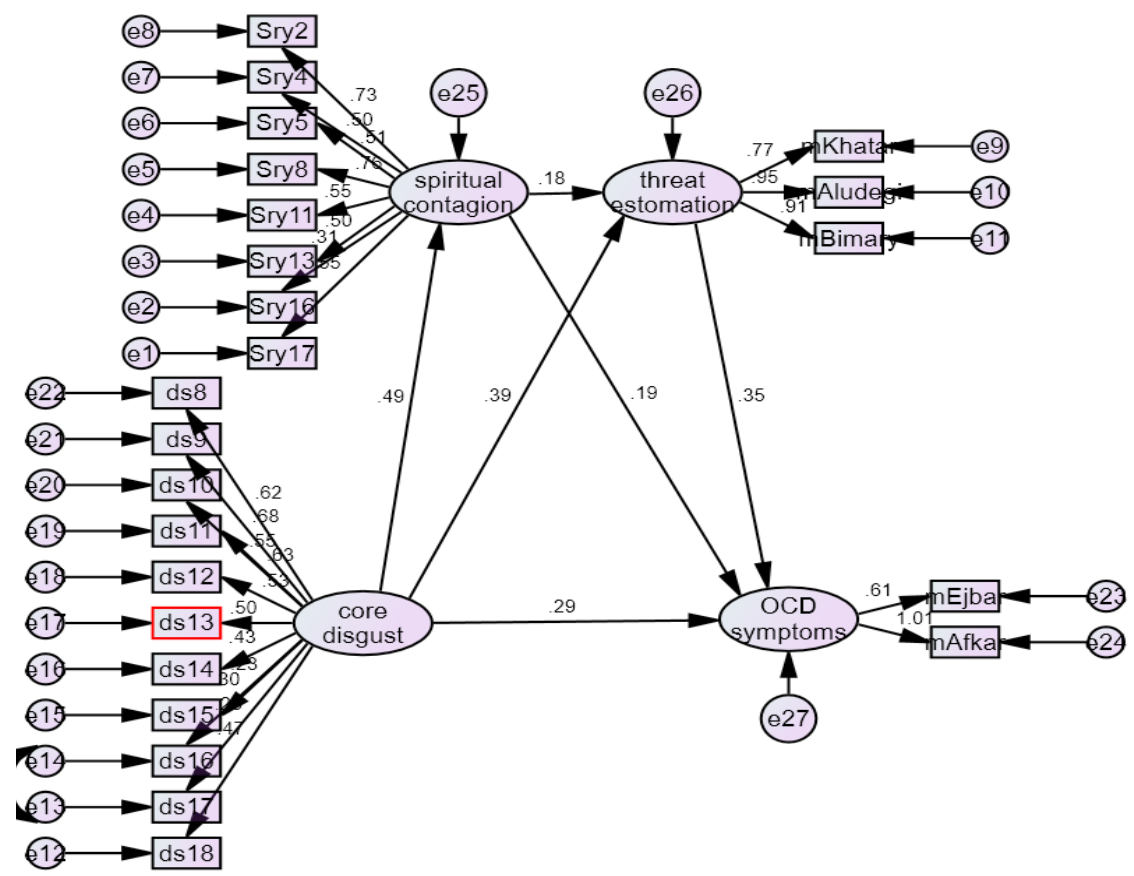

Iranian Journal of
PSYCHIATRY AND CLINICAL PSYCHOLOGY

Figure 1. The structural model of the relation between disgust and OCD symptoms with the mediation roles of spiritual contagion and threat estimations

avoidance of objects such as door handles can be explained by the perception of a "chain of contagion" in these patients, which means that they can detect the contamination track to further points from the original source, without decreasing in the original amount of contamination.

In another research Zanjani et al. [31] found that a related concept to the law of contagion means looming vulnerability to contamination mediated the relation between disgust and fear of contamination which showed that people with OCD perceived contaminated stimuli not as fixed but as spreadable, approaching to them and in a way that the threatening force of them is increasing. In the same way, in the present study, the mediation role of the law of contagion showed that people with contamination-based OCD believed that through a brief contact a considerable amount of contamination or the "essence" can transmit from a contaminated object to the neural one and this essence remains for a long time.

In the law of contagion, the transmission of pollution is not like a layer that can cover a surface of something but it is the essence transmission that may cause the nature of something to get changed. It is obvious that if one perceived that the essence of something has changed, even repeated washing cannot convert it, so this can explain the overestimations of threat and frequent washing in people with OCD. This overestimation of threat is consistent with Cisler et al. [32] that showed an overestimation of danger from obsessional beliefs can moderate the relation between disgust and fear of contamination. Another part of negative spiritual contagion things are related to the other function of disgust means protection of the psychological body [9].

Our results showed that people with the fear of contamination attempt to protect themselves from the transmission of immoral, behavioral or ominous characteristics. This concept of moral or spiritual contamination as Fedotova [23] has mentioned, is different from Rachman's mental pollution [34]. The role of the law of contagion in OCD which cannot be explained by logical accounts is consistent with the previous research showed the role of magical thinking in OCD such as Thought-action Fusion beliefs [35] and magical ideation [36]. Finally, as researchers suggested, the law of contagion is intuition or heuristic [38], which its mediational role may have important implications in OCD treatment.

\section{Ethical Considerations}

\section{Compliance with ethical guidelines}

After explaining the study Objectives to the participants and assuring them of the confidentiality of their information and being free to leave the study at any time, an informed consent was obtained from them. This study has an ethical approval. 


\section{Funding}

This study was extracted from the $\mathrm{PhD}$. thesis of first author approved by the Department of Clinical Psychology at Shahed University.

\section{Authors contributions}

Conceptualization, writing, editing \& review: Mona Moradi Motlagh, Mohammadreza Nainian, and Ladan Fata; Methodology and software: Mohammad Gholami and Mona Moradi Motlagh; Data curation: Mona Moradi Motlagh and Gholamhosein Ghaedi.

\section{Conflicts of interest}

The authors declare no conflict of interest. 
This Page Intentionally Left Blank 


\section{مدل ساختارى رابطه ميان جندش و نشانههاى وسواس آلودتى با نقش ميانجى قانون سرايت و برآوردهاى تمهديد}

مونا مرادى مطلق' (1)، "محمدرضا نائينيان' (1)، لادن فتى'، محمد غلامى فشاركى"، غلامحسين قائدى '

1. كروه روانشناسى بالينى، دانشكده علوم انسائى، دانشعاه شاهد، تهران، ايران.

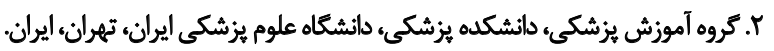

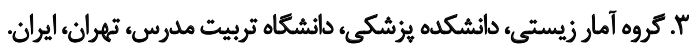

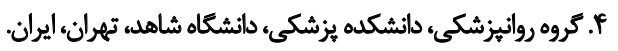

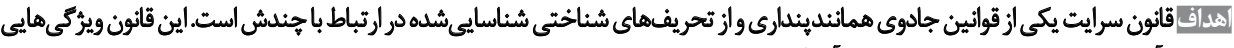

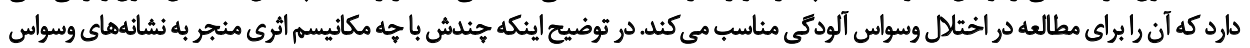

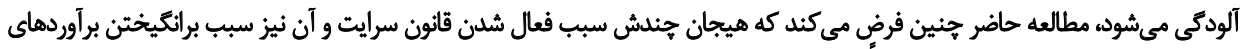

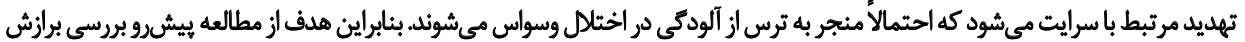

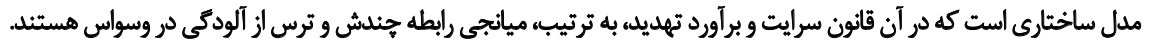

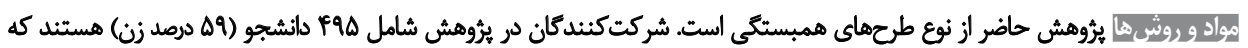

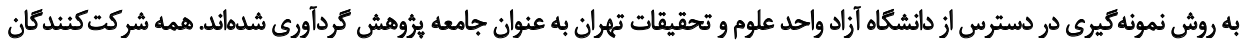

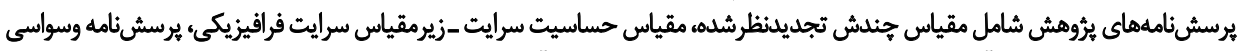

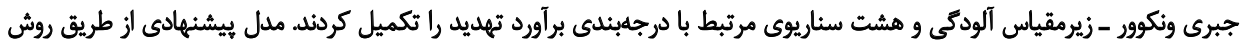
مدل سازى معادلات ساختارى (SEM) و با نرمافزال

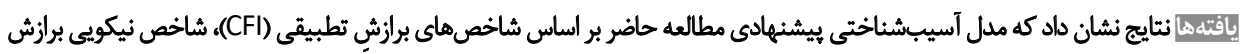

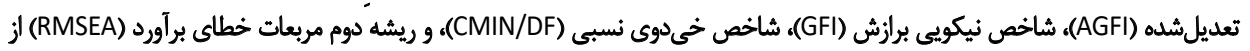

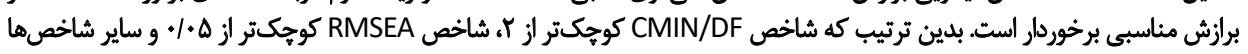

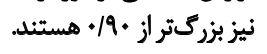

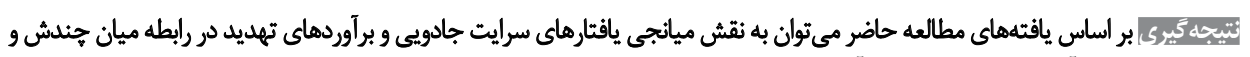

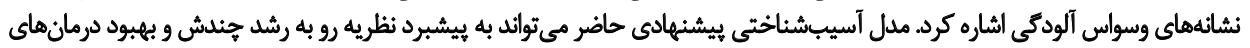
شناختى -رفتتارى مرتبط با آن كمك كند.

تاريخ دريافت: P|خرداد

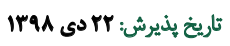

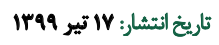

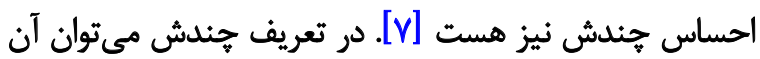

dales

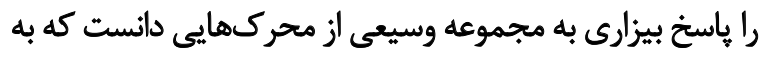

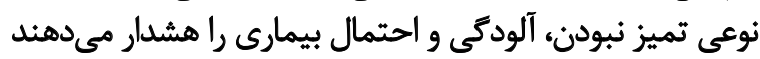

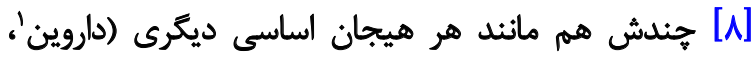

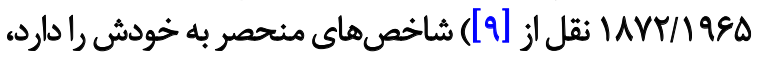

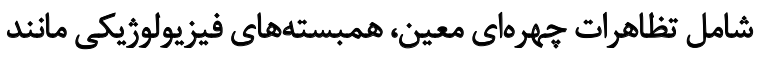

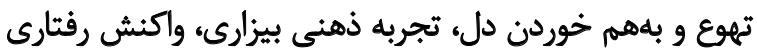

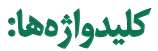

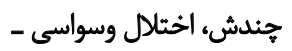

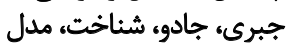

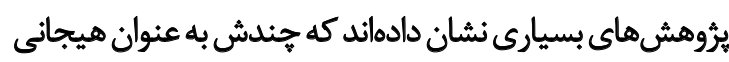

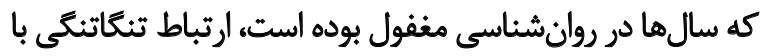

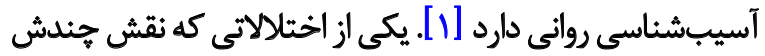

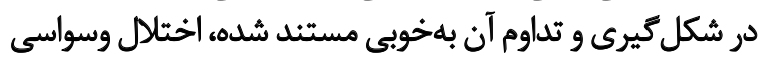

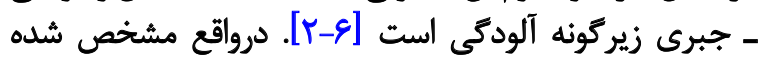

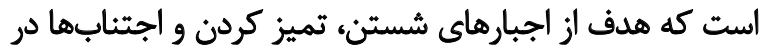

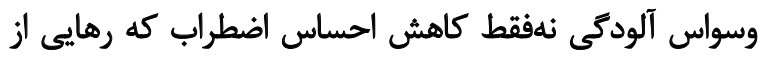




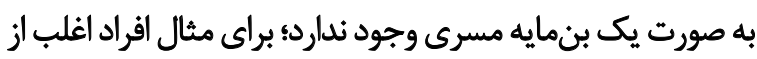

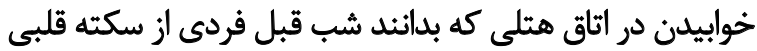

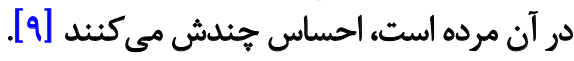

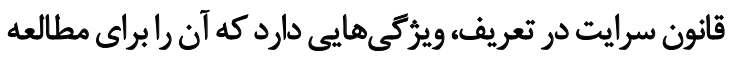

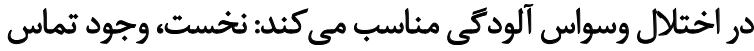

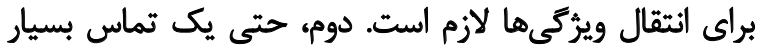

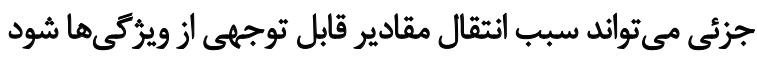

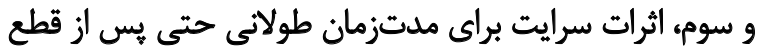

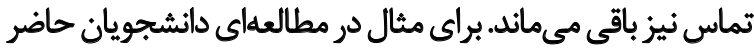

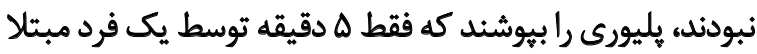

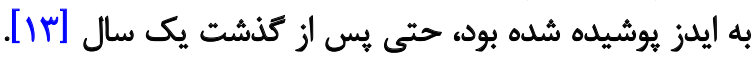

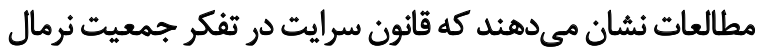

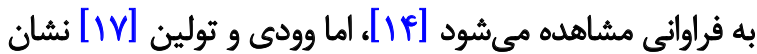

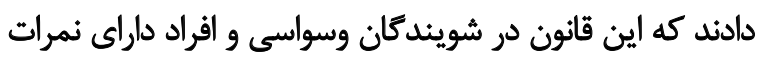

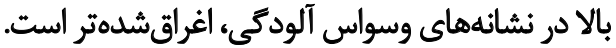

ادراك سرايت به صورت اغراقشده سبب مى إشود كه افراد، به

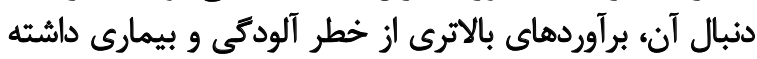

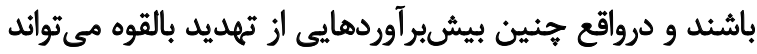

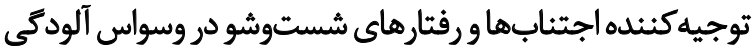

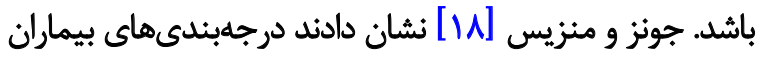

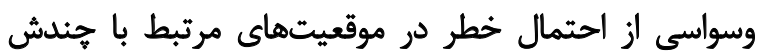

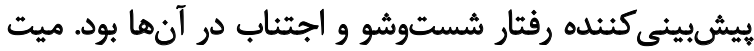

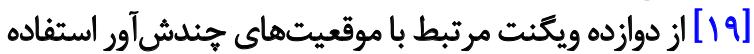

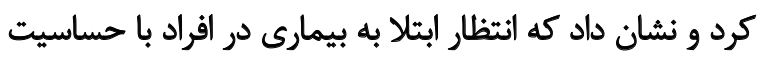

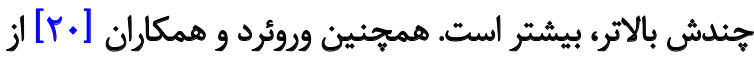

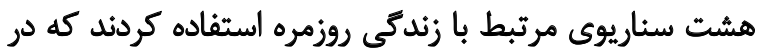

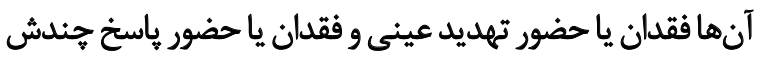

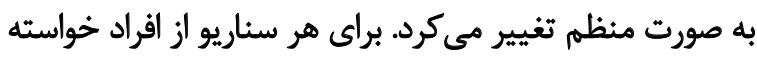

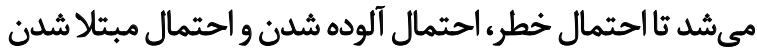

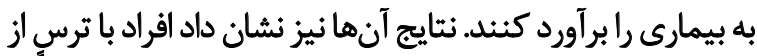

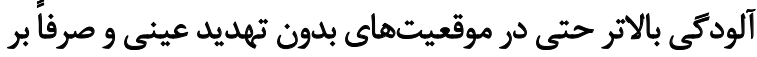

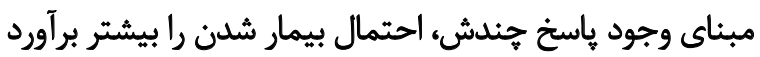

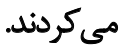

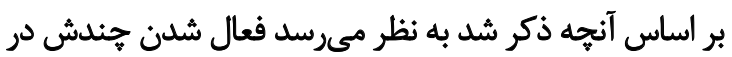

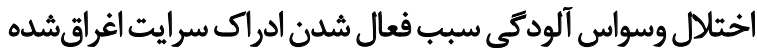

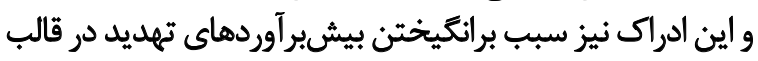

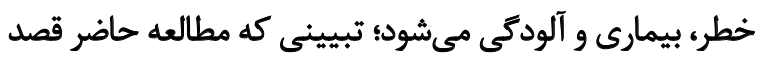

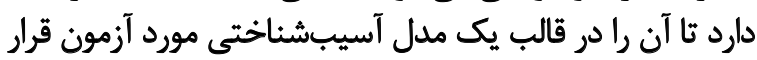

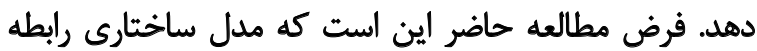

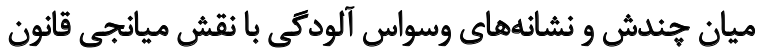

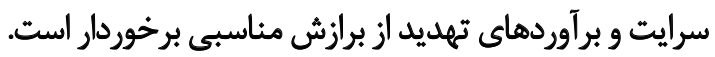

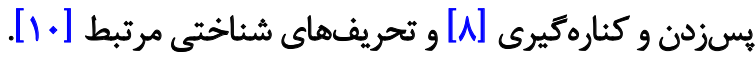

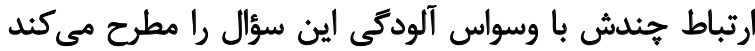

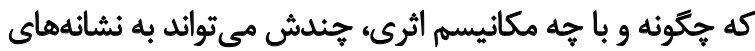

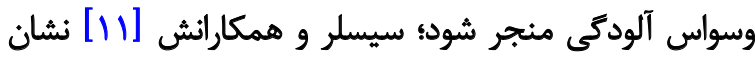

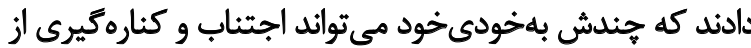

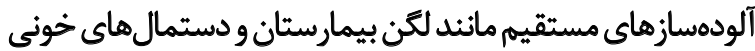

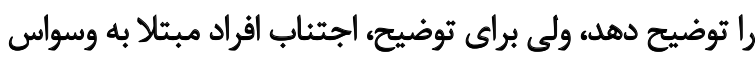

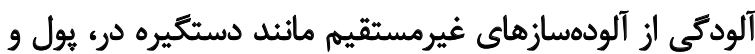

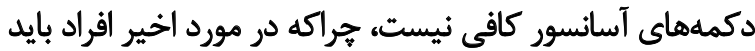

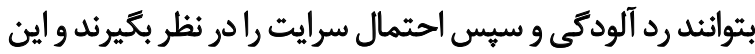

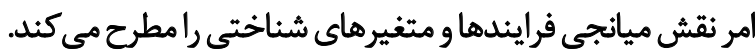

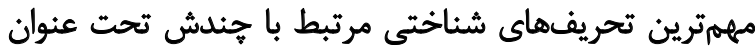

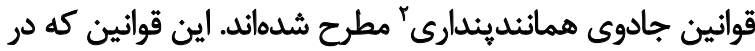

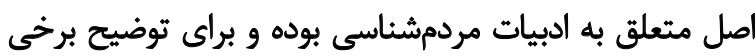

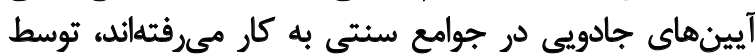

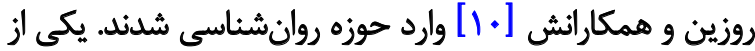

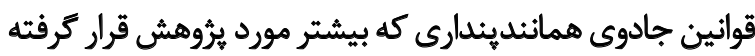

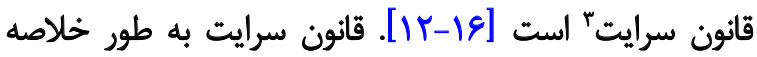

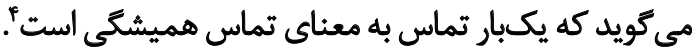

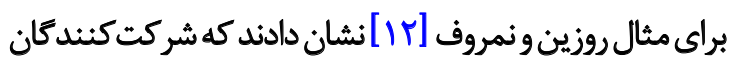

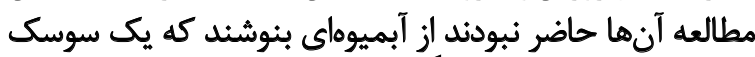

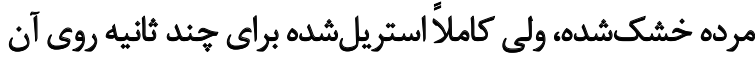

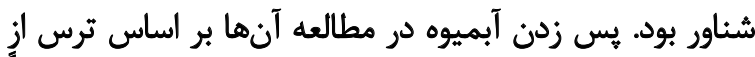

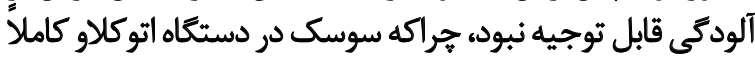

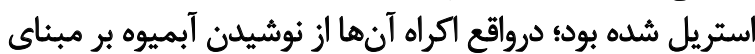

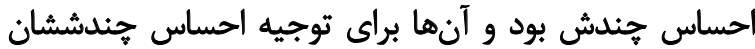

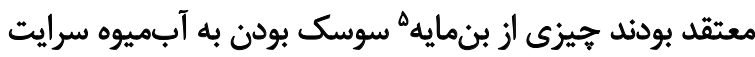

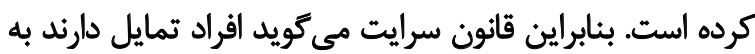

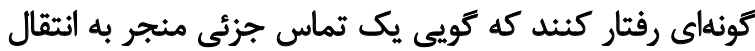

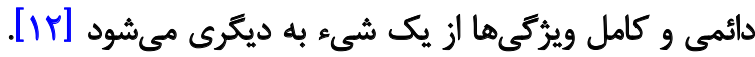

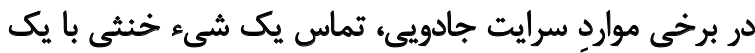

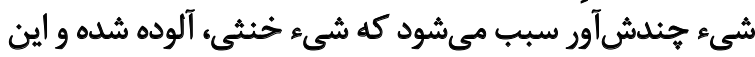

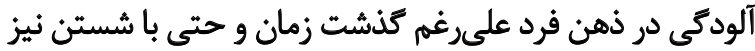

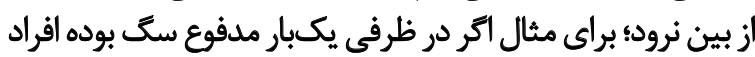

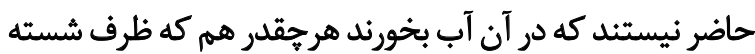

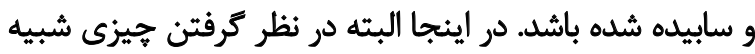

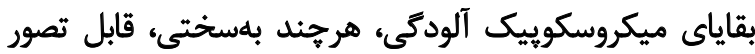

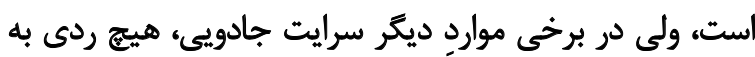
صورت مادى قابل شناسايى نيست؛ يعنى هيج معادل فيريكي جيكي

2. Sympathetic magic

3. Low of contagion

4. Once in contact, always in contact

5. Essence 


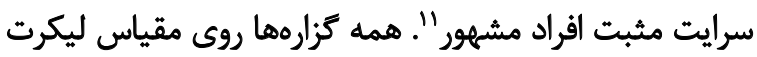

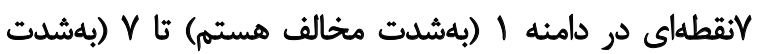

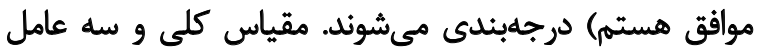

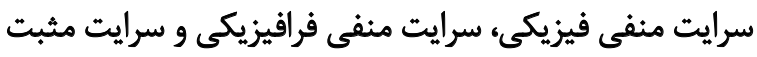

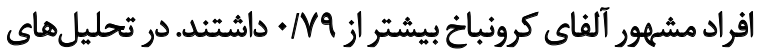

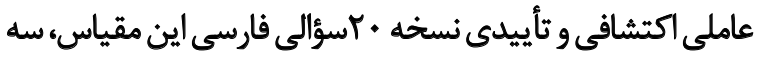

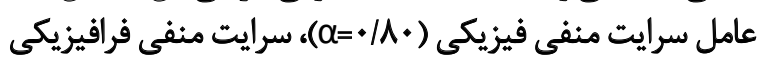

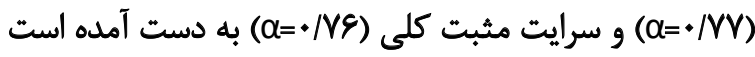
كه در اين مطالعه فقط از زيرمقياس سرايت منفي

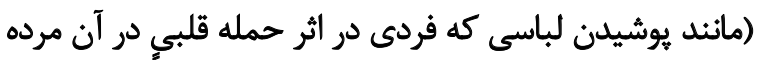

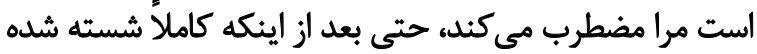
باشد) كه منطبق با تعريف قانون سرايت استه استفاده شده است.

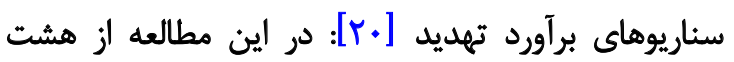

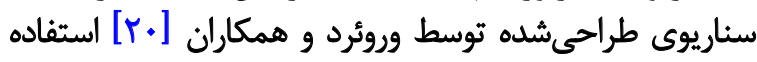

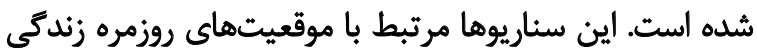

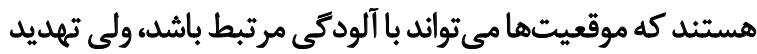

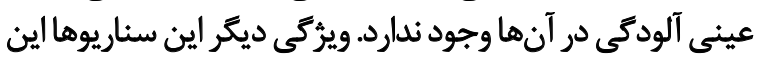

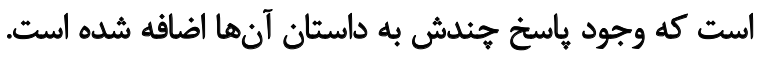

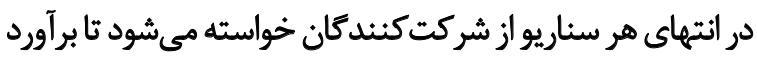

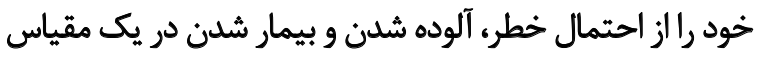

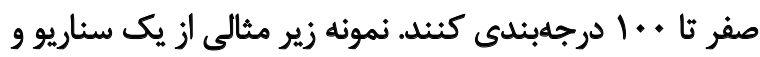

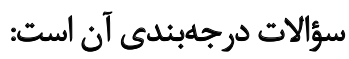

شما از توالت بيرون مىآييد. شير آب را باز مىكنيد و و

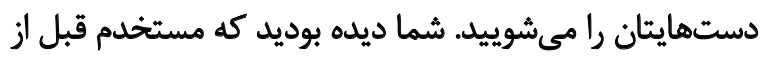

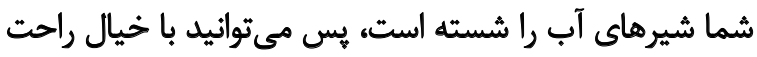

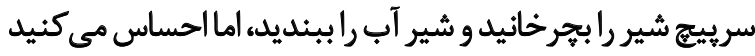

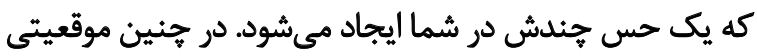

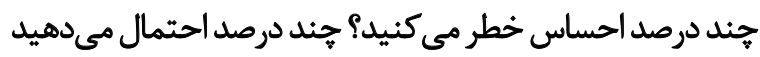

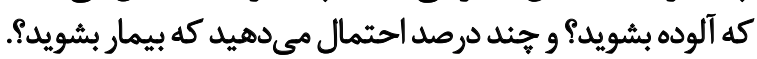
براى ارزيابى ويزگكى هاى روانسنجى سناريوهاى برآورد تهديد

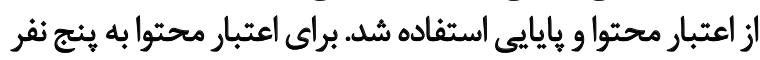

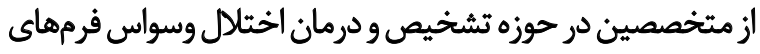

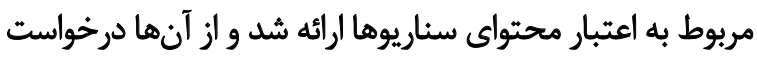

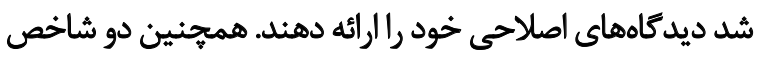
لاوشه

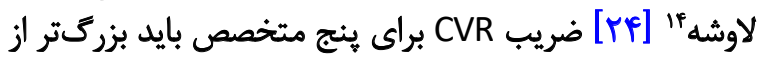

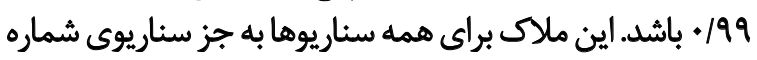

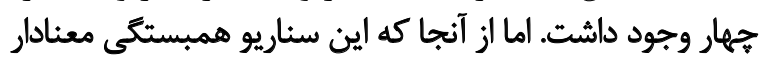

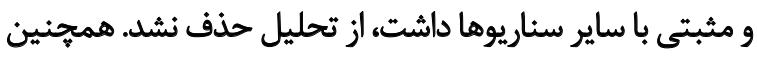

11. Positive Celebrity Contagion

12.Content Validity Ratio

13. Content Validity Index

14. Lawshe
اين مطالعه از نوع طرحهاى مقطعى ـ همبستكى است. جامعه

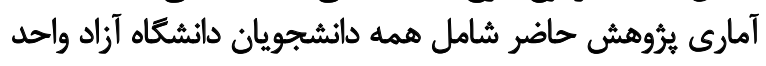

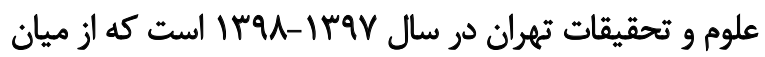

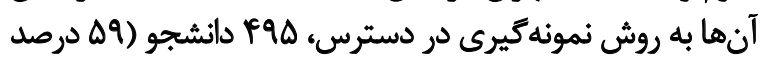

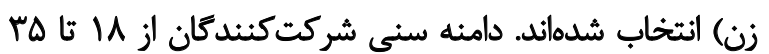

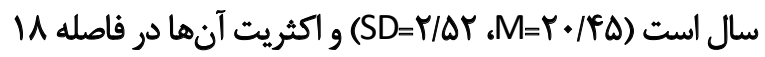
تا • r سال قرار دارند (\$F/F درصد)

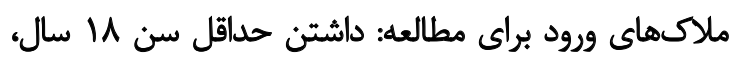

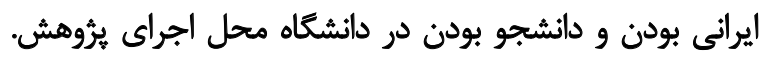

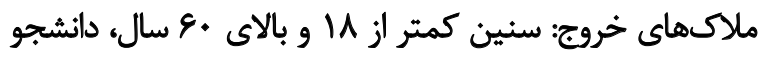

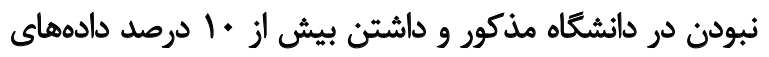

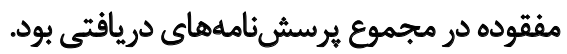

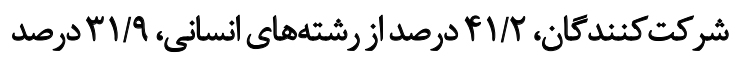

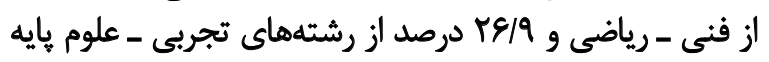

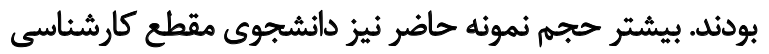

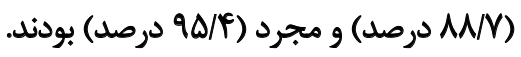

إبزازأها

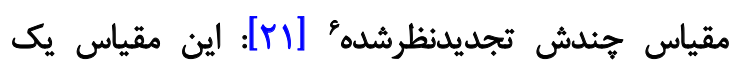

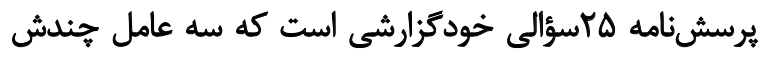

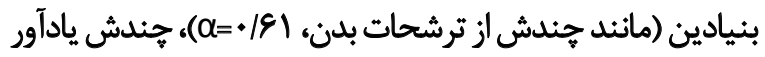

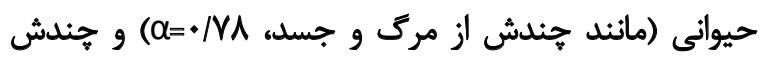

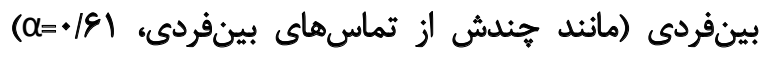

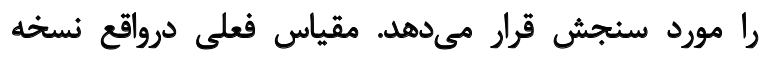

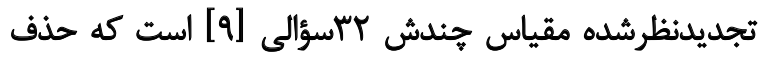

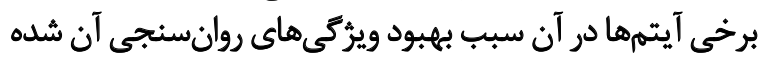

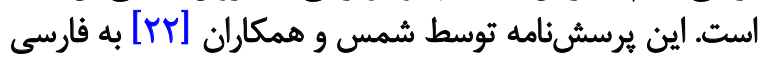

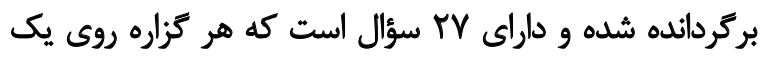

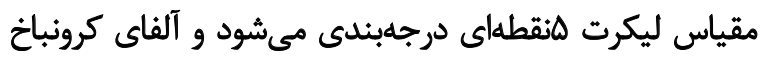

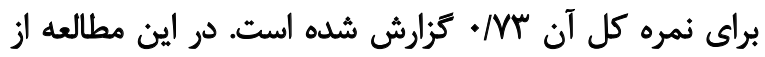

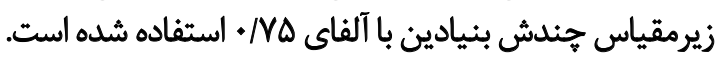

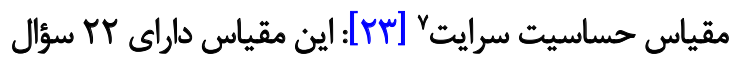

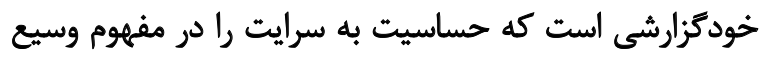

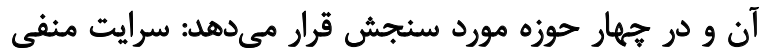

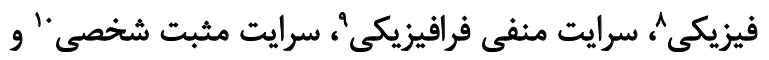

\footnotetext{
6. Disgust Scale-Revised (DS-R)

7. Contagion Sensitivity Scale (CSS)

8. Negative Physical Contagion

9. Negative Spiritual Contagion

10. Positive Personal Contagion
} 
عنوان شاخص هاى توصيفى برايى متغيرهاى مورد مطالعه محاسبه

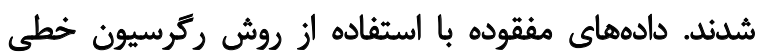

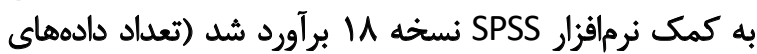

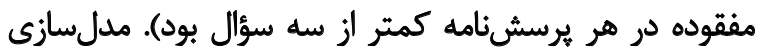

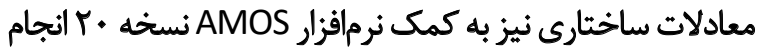

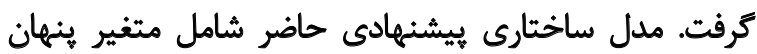

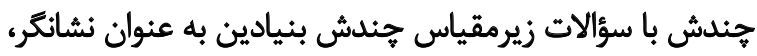

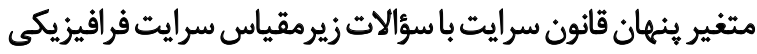

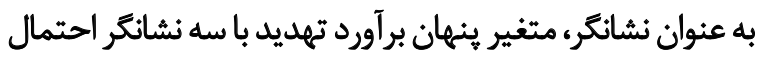

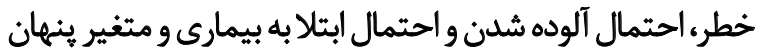

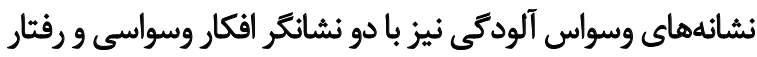

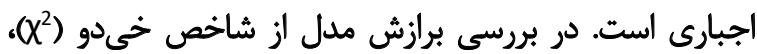

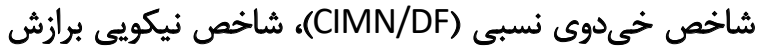

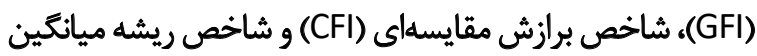

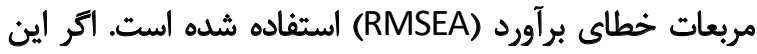

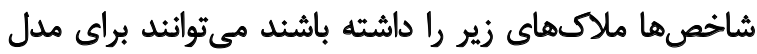

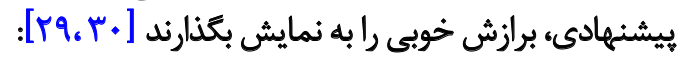

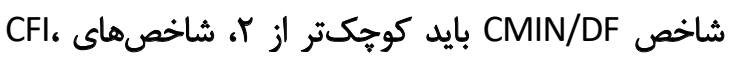
GFI و AGFI كوجكتر از ه+٪ بـ باشند.

يأقتهها

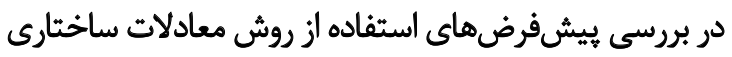

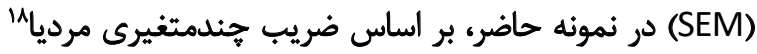

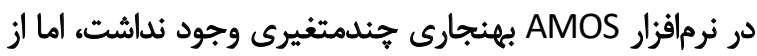

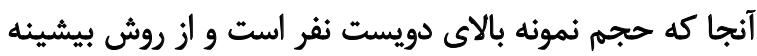

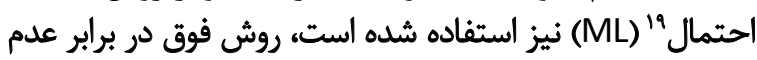

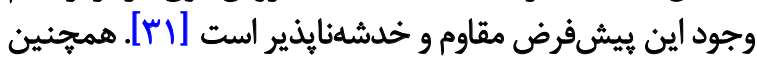

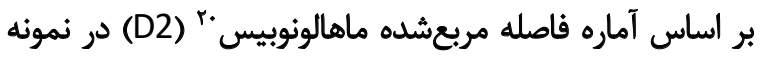

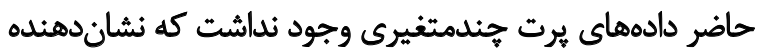

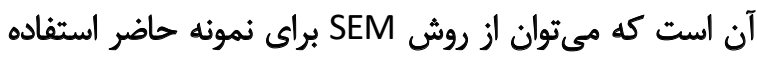

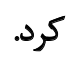

ميانكين (انحراف استاندارد) و ضرايب همبستكى ويرسون

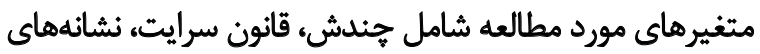

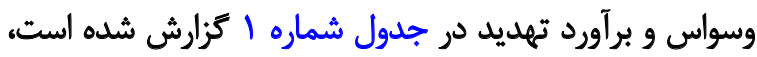

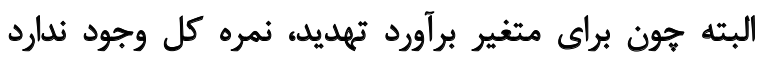

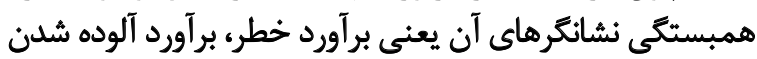

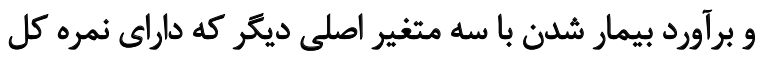

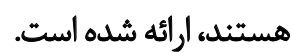

18. Mardia

19. Maximum likelihood

20. Mahalanobis

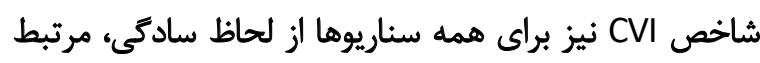

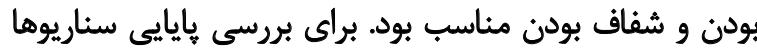

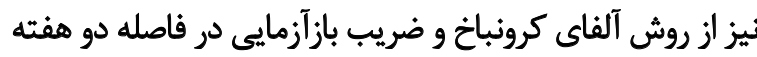

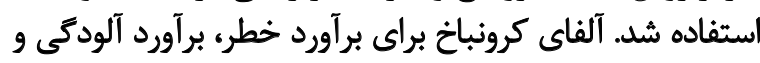

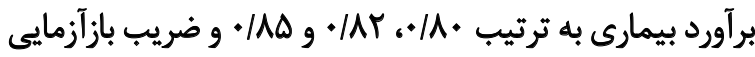

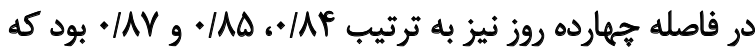

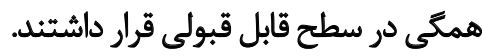

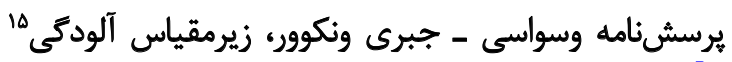

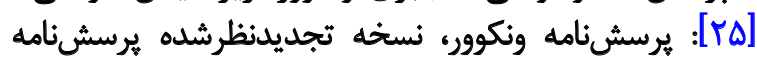

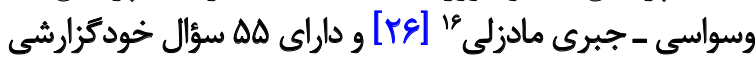

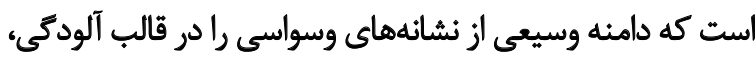

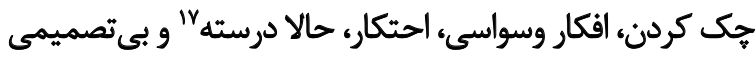

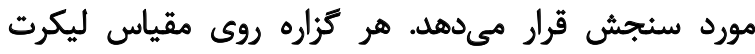

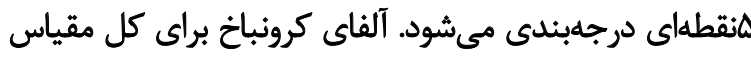

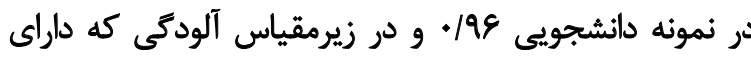

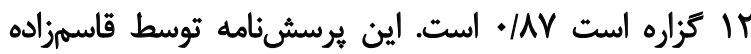

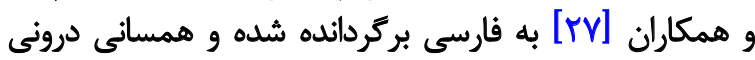

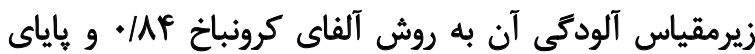

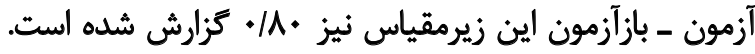

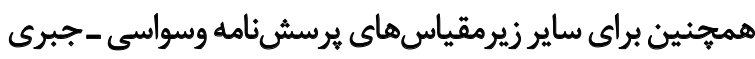

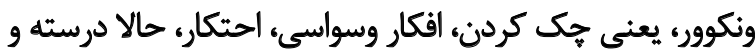

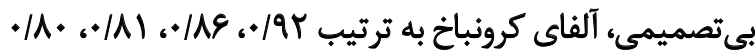

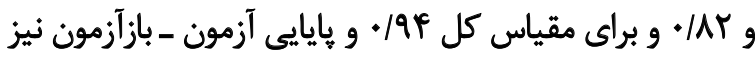

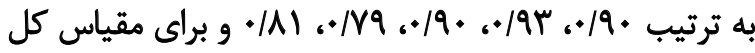

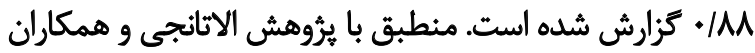

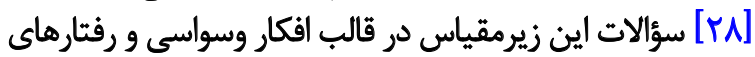
اجبارى مورد بررسى قرار مي زيريرد.

روش إيرا.

كردآورى نمونه در مطالعه حاضر بدين طريق انجام شد كه

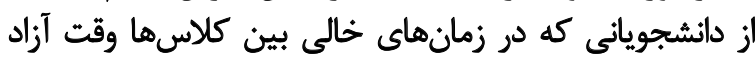

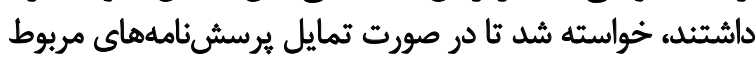

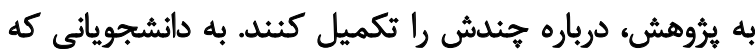

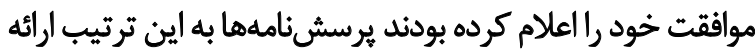

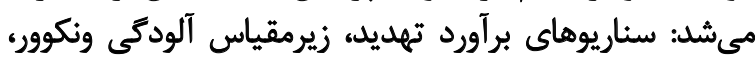

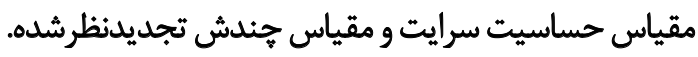

روش تحليل دادهها

ميانكين، انحراف استاندارد و ضرايب همبستكى يُيرسون به

15. Vancouver Obsessional Compulsive Inventory-Contamination subscale (C-VOCI)

16. Maudsley

17. Just right 
جدول ا. ميانكين (انحراف استانداره) و ضرايب همبستكى متغيرهاي مدل

\begin{tabular}{|c|c|c|c|c|c|c|c|}
\hline مياتكين 土|نحراف أستارد & نشائهاى وسواس & ئدش & سرايت & برآورد بيمار شدن & برآورد آلوده & برآورد خطر & \\
\hline IV/FVIIF/Mr & .10 &.$/ m$ &.$/ K A$ & 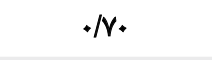 & $\cdot M r$ & 1 & برآورد خطر \\
\hline Tr/TrIIF/qه & $\cdot / \Delta r$ &.$/ 4$ &.$/ r$ & $.1 N E$ & 1 & & برآورد آلوده شدن \\
\hline WrotIr/MP & . IF &.$/ 19 q$ &.$/ r$ & 1 & & & برآورد بيمار شلن \\
\hline$r / q Y \pm V / \cdot r$ &.$|p|$ &.$/ \% \Delta$ & 1 & & & & قانون سرايت \\
\hline$r / P \cdot \pm \cdot / M^{\prime}$ & . $/ P \varphi$ & 1 & & & & & جندش \\
\hline$V / F V \pm / M$ & 1 & & & & & & تشانهذاى وسواس \\
\hline
\end{tabular}

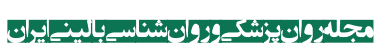

جدول r. اثرات مستقيه، غيرمستقيهم وكل و معنادارى آنها در روابط بين متغيرهاى مدل

\begin{tabular}{|c|c|c|c|}
\hline أثر غير مستقيم & اثر مستقيم & 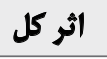 & 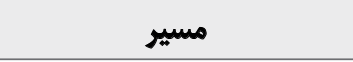 \\
\hline- &.$/ 419$ &.$/ 419$ & از جندش به قانون سرايت \\
\hline $.1 \cdot 18$ & . HAV &.$/ F f^{2}$ & از جندش به برآورد تهديد \\
\hline . MAF & . rar & . IAfE & از جندش به نشانههاى وسواس \\
\hline- & $.11 \mathrm{~W}$ &.$/ 1 \mathrm{~W}$ & از قانون سرايت به برآورد تهنديد \\
\hline .1 .81 & $.1 N$ &.$/ M e$ & از قانون سرايت به نشائههاي وسواس \\
\hline- &.$/ M e \Delta$ &.$/ M P Q$ & از برآورد تهديد به نشائهاي وسواس \\
\hline
\end{tabular}

آمد:

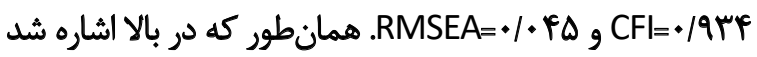
بر اساس ملاكهاى تعيينشده، مدل حاصل، از برازش مناسبى

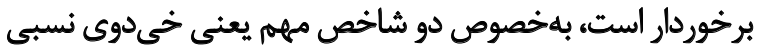

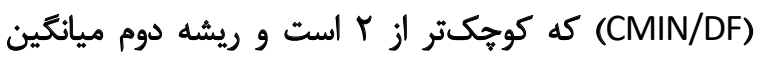

مدل بيشنيهادي رابطه ميان هندش و نشانههاي وسواس

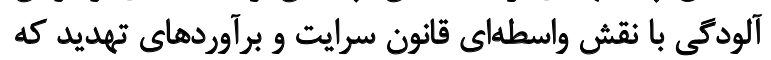

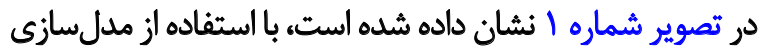

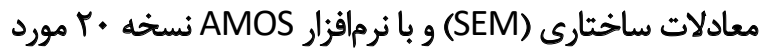

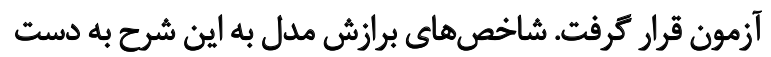

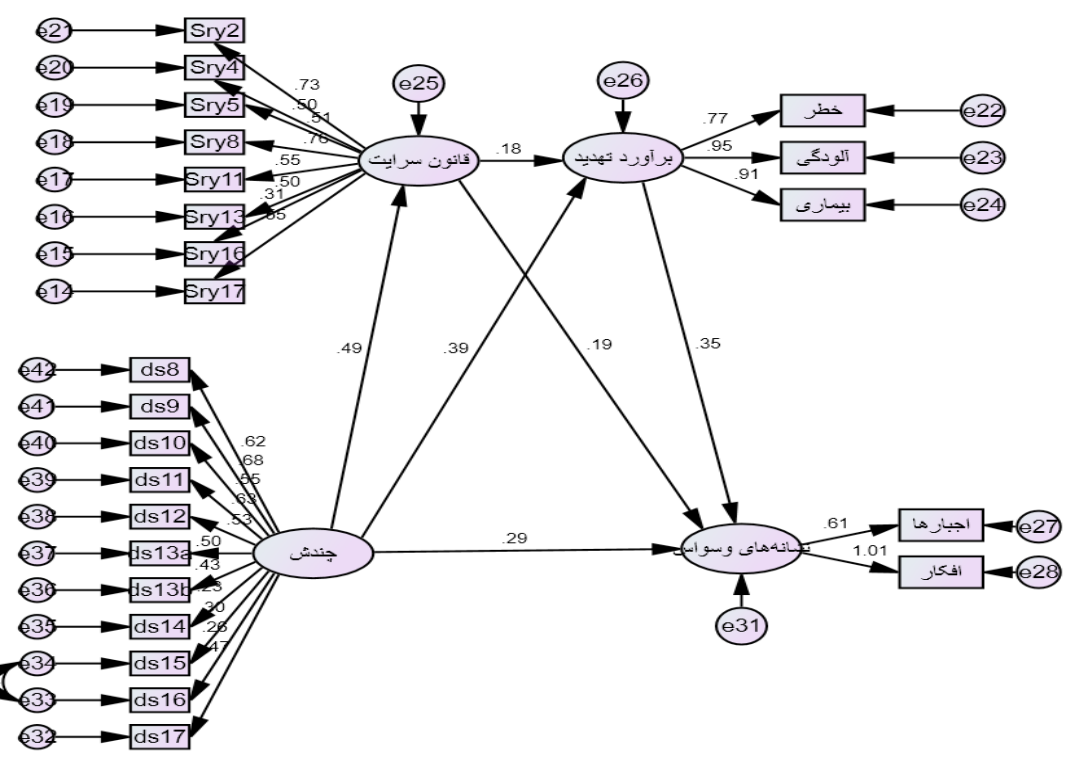

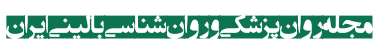

تصوير ا. مدل ساختارى رابطه ميان جندش و نشانهاى وسواس آلودكى با نقش واسطهاي قانون سرايت و برآوردهاى تهديد و ضرايب استاندارد آنها 


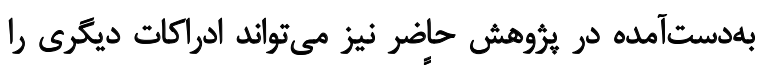

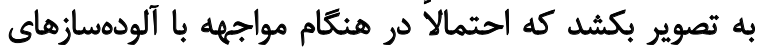

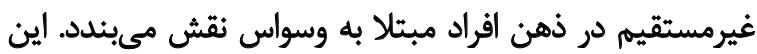

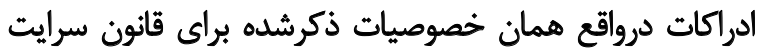

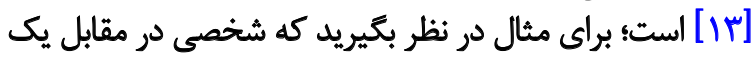

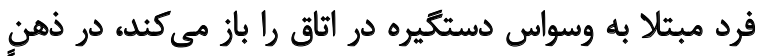

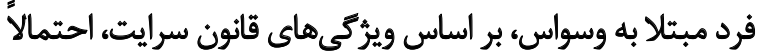
اين ادراكات اتفاق مى افتدا: 1.

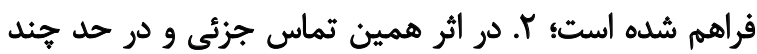

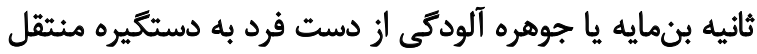

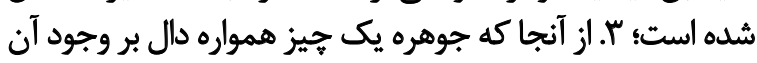

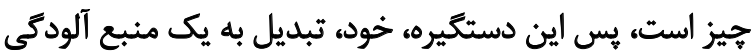

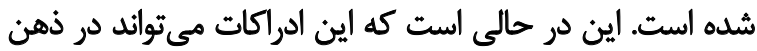

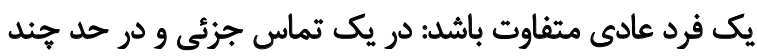

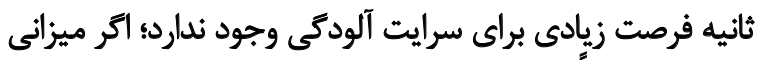

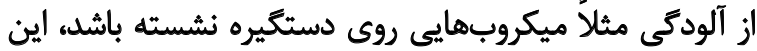

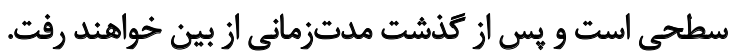

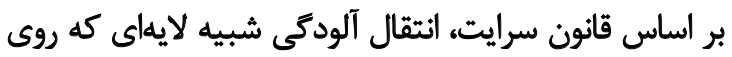

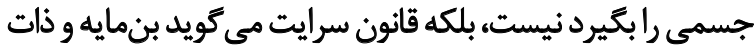

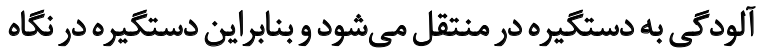

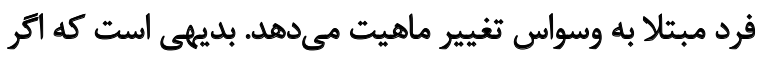

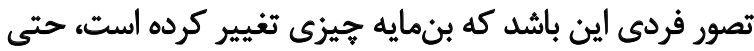

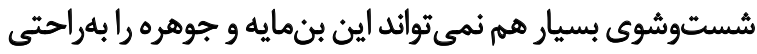

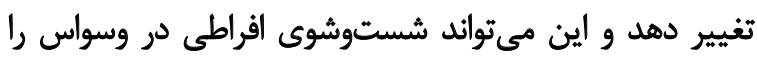

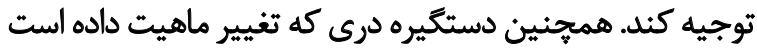

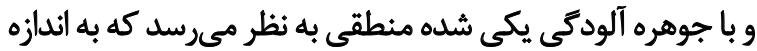

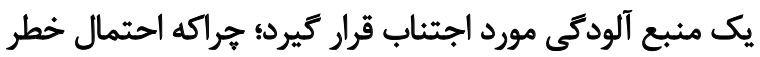

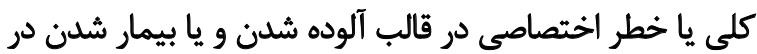

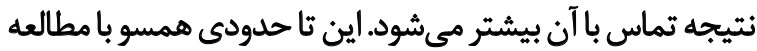

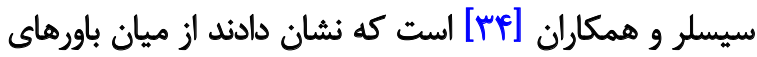

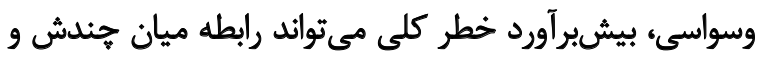

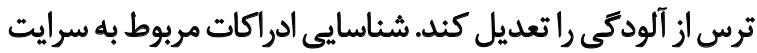

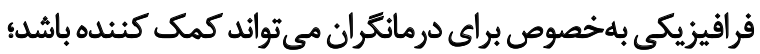

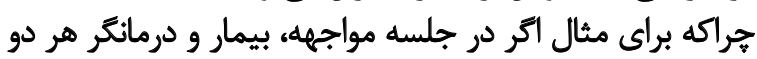

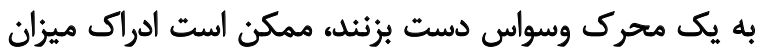

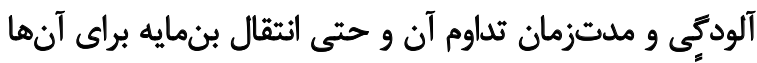

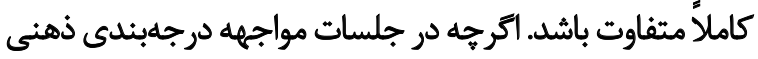

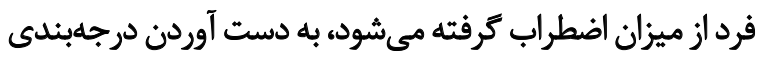

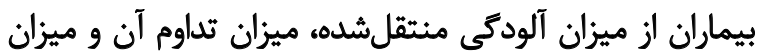
تغيير ماهيت در شىء مقصد نيز ممكن است مفيد باشيد.

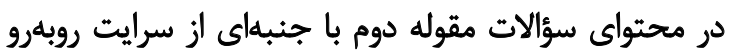

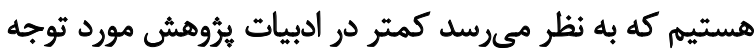

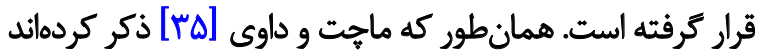

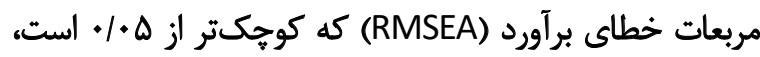

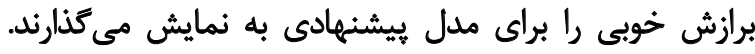

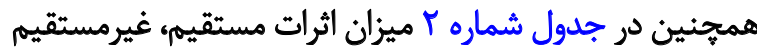
و كل و معنادارى آنها در روابط بين متغيرهاى مدان مدل ارائه شده

Ho

يُروهش حاضر با استفاده از مدلسازى معادله ساختارى

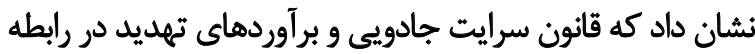

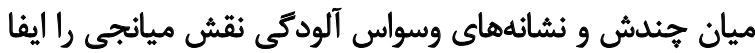

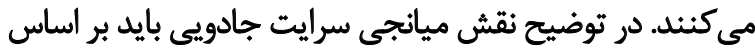

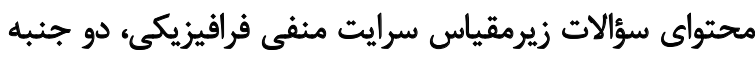

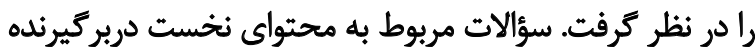

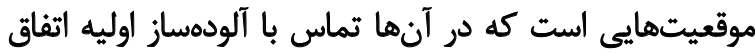

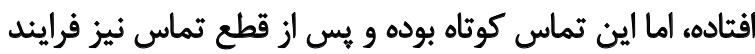

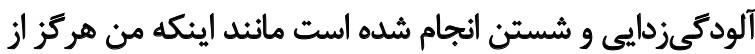

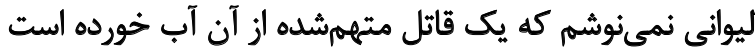

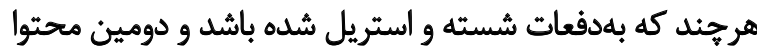

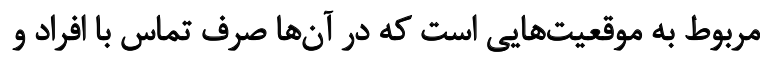

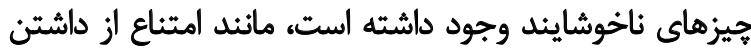

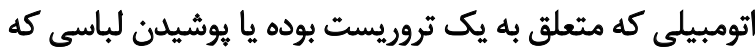

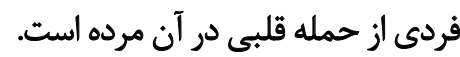

دمتواى نخست مي تواند تا حدودى نقش ميانجى خصوصيات

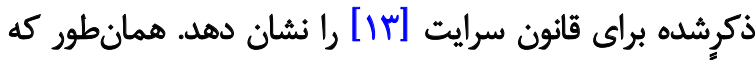

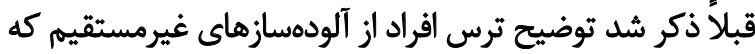

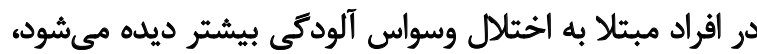

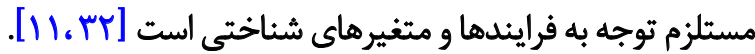

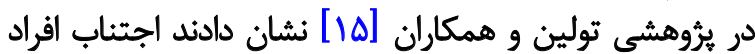

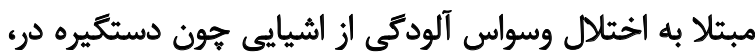

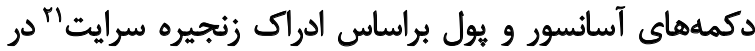

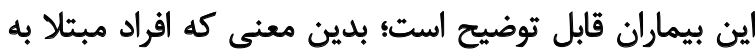

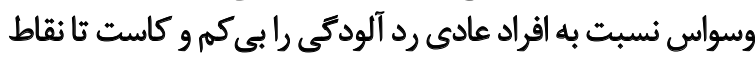

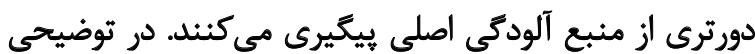

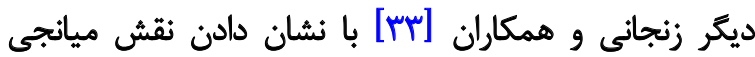

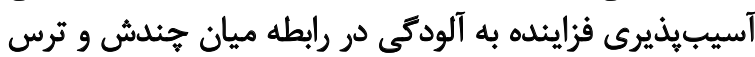

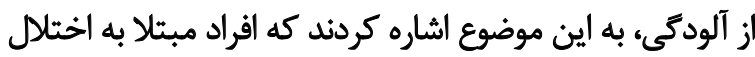

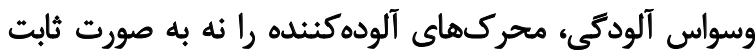

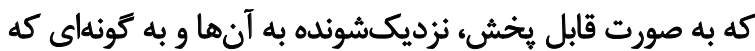

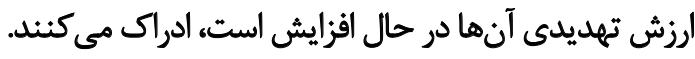
در ادامه شناسايى نقش ميانجى جنين ادراكاتى در رابطه جندش و نشانههاى وسواس آلودگى، نقش واسطهائ سرايت جادويى

21. Chain of contagion 


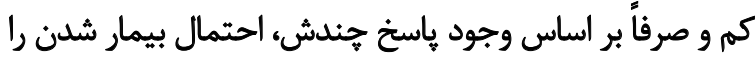
به طور معنادارى بيشتر برآورد مى كنيند.

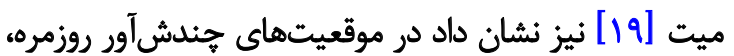

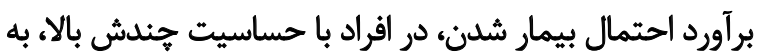

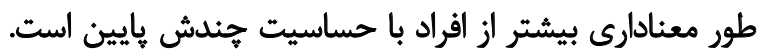

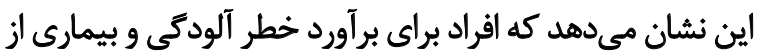

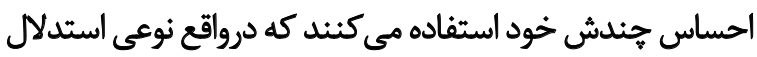

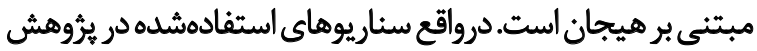

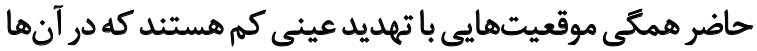

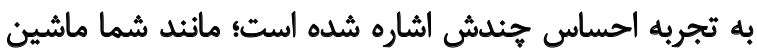

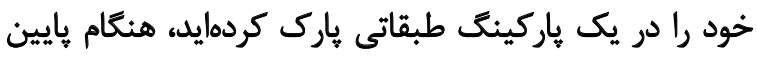

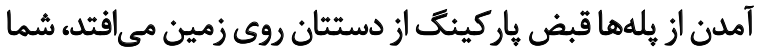

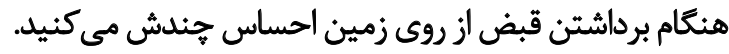

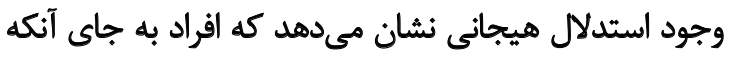

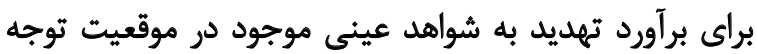

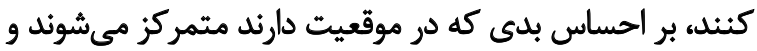

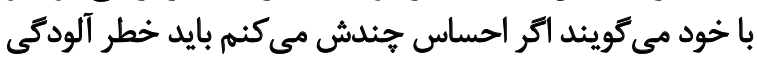

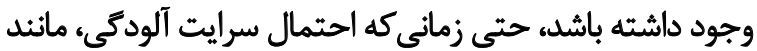

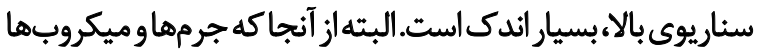

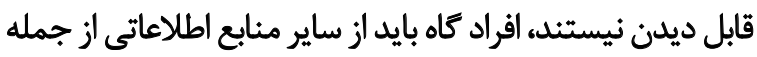

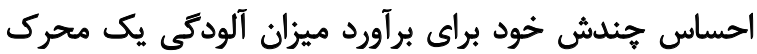

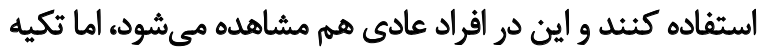

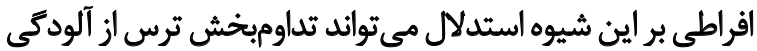
در اختلال وسواس باشد [ب. [ب.

نكته مهمى كه درباره هر دو تحريف شناختى فوق وجود دارد

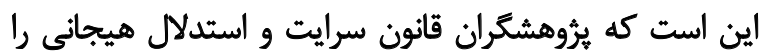

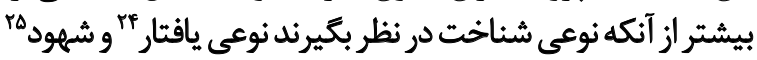

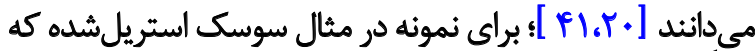

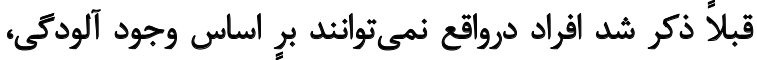

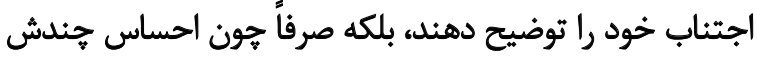

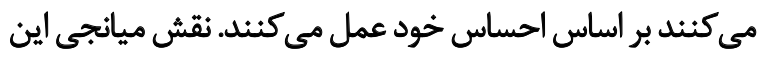

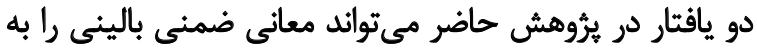

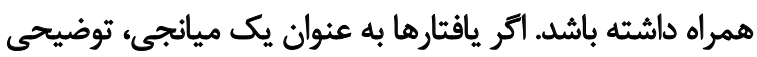

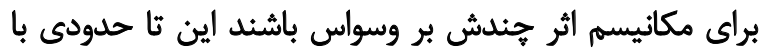

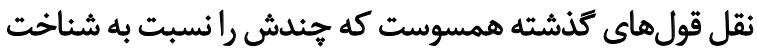

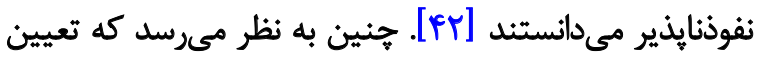

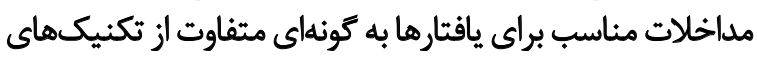

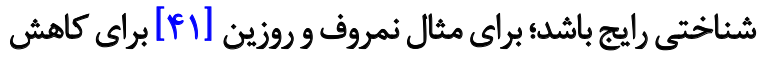

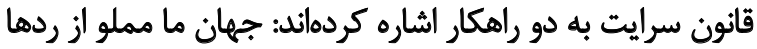

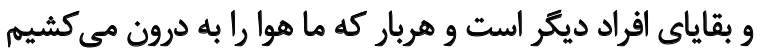

24. Heuristic

25. Intuition
كاركرد عمده جيندش محافظت از بلدن در برابر آلودگى و بيمارى

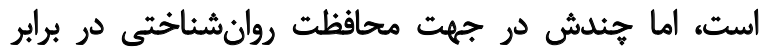

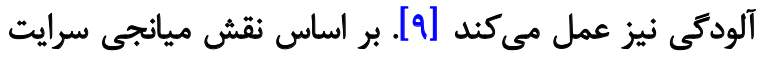

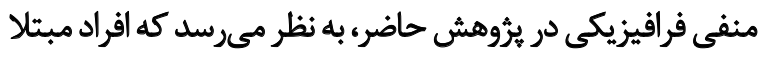

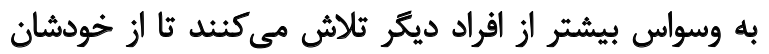

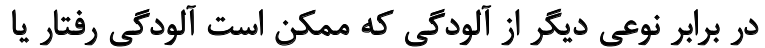

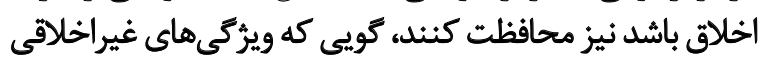

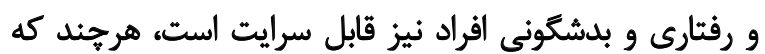

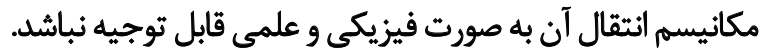

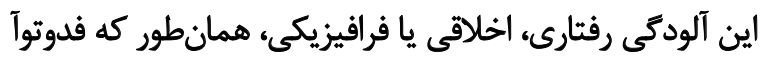

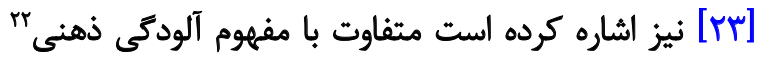
راجمن [عب] استاره

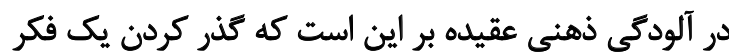

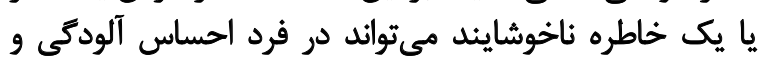

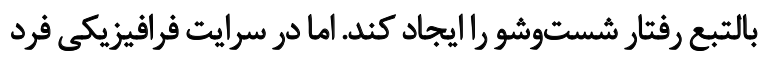

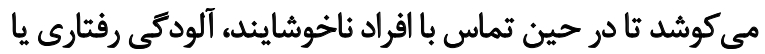

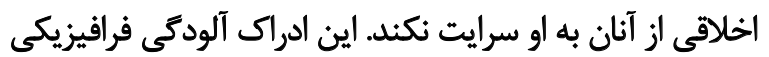

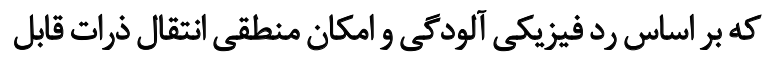

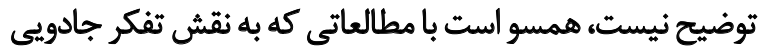

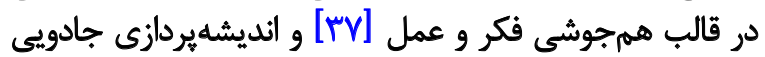

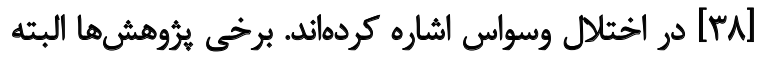

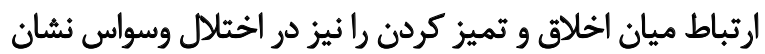

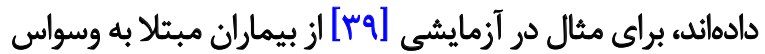

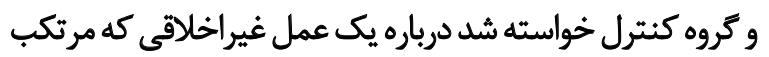

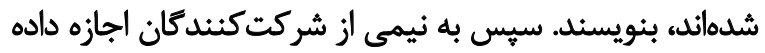

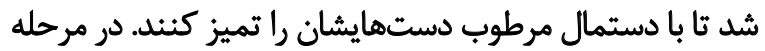

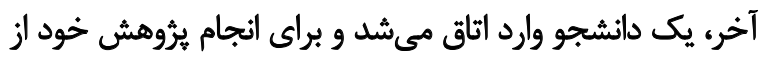

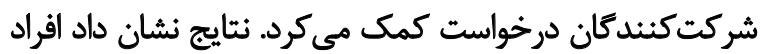

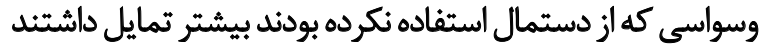

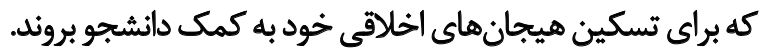

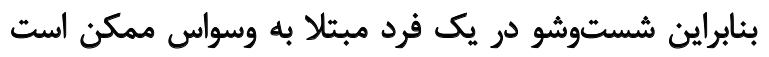
در اثر احساس سرايت جيزى غيراخلاقيى و يا بائ براي فرونشاني هيجانهاى اخلاقى نيز انجام شود.

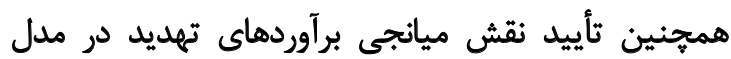

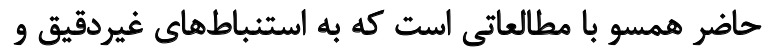

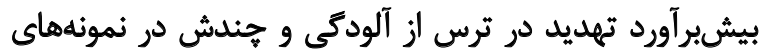

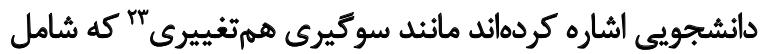

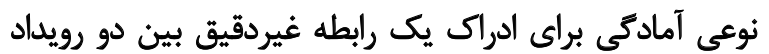

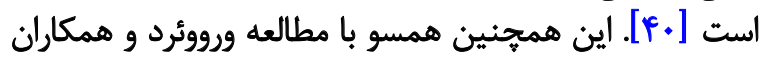

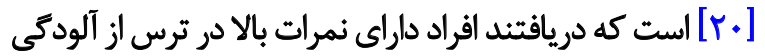

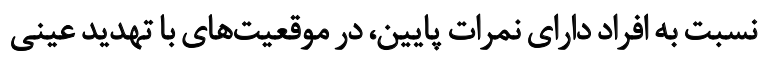


نمونه بالينى در صورت امكان مىتواند مفيد باشد. هرجند درا ماري

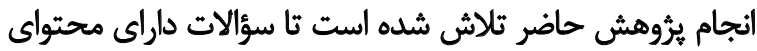

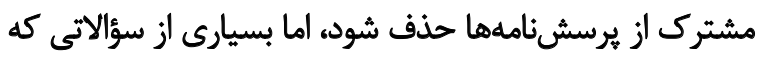

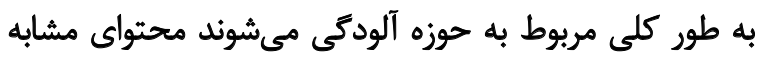

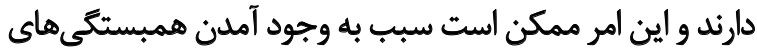

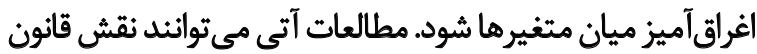

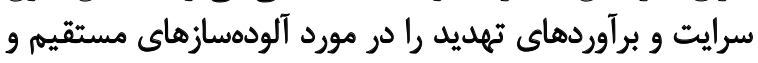

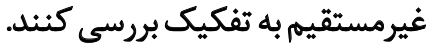

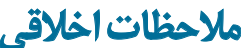

\section{بيروى از اصول اخغلاق يثوهش}

براى دانشجويان درباره هدف مطالعه و فعاليتى كه بايد

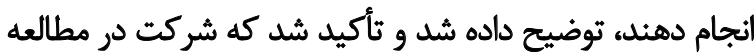

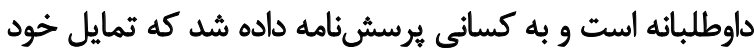

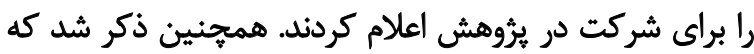

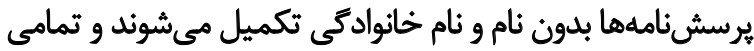

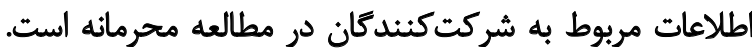

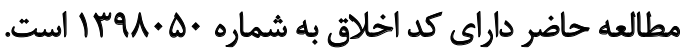

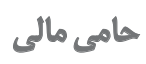

مقاله حاضر بركرفته از رساله دكتراي نويسنده اول (مونا مرادى مطلق) در كروه روانشناسى بالينى دانشكاه شاهد است.

$$
\text { هشاركت نويسيند مكان }
$$

مفهومسازى، نكارش، ويراستارى و اصلاحات: مونا مرادى

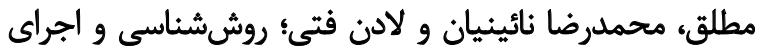

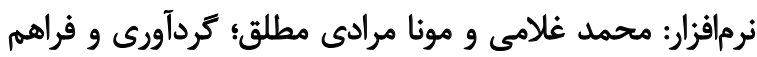

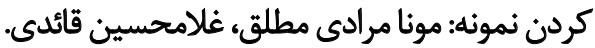

$$
\text { تعارض مثاقع }
$$

اين يُروهش تعارض منافع نداشته است.
اين احتمال هست كه آن هوا دربركيرنده مولكول هايي باشد كه

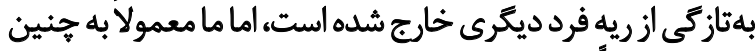

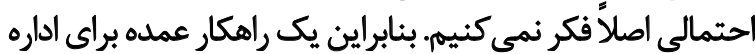

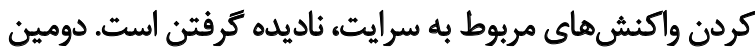

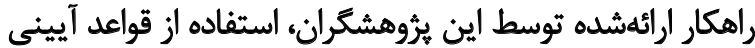

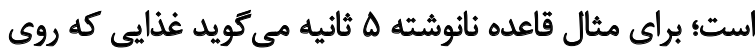

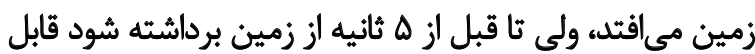

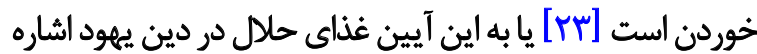

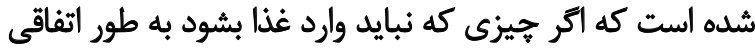

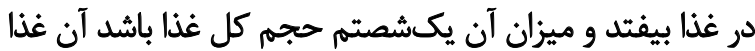

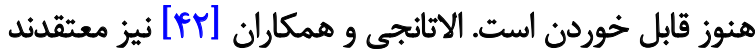

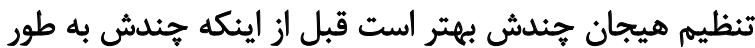

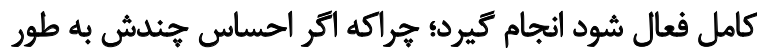

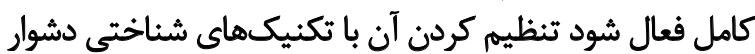

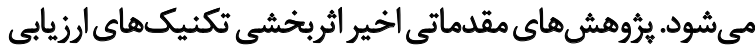

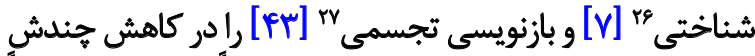

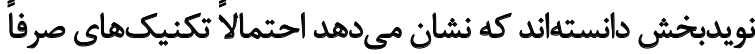

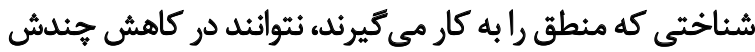

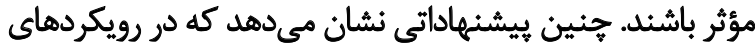

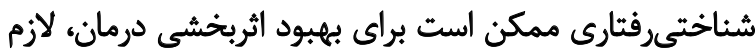

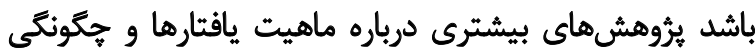
تغيير آنها بهخصوص در اختلال وسواس انجام شود.

$$
\text { نتيجهَيرى }
$$

در يايان، مطالعه حاضر نشان داد كه قانون سرايت جادويى و

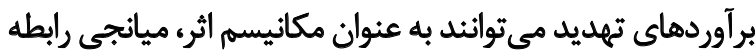

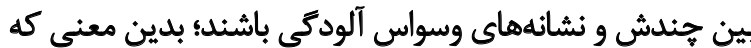

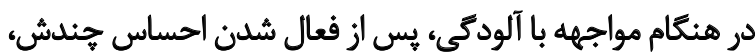

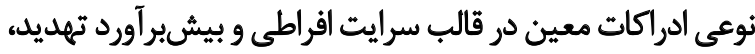

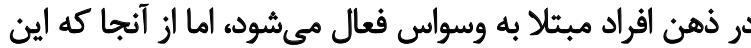

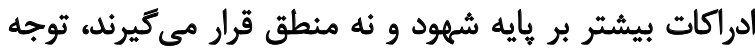

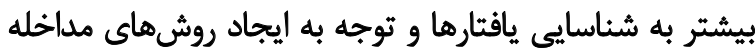

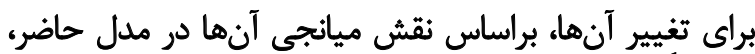

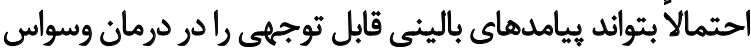

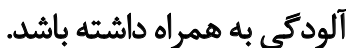

ثرؤش حاضر محدوديتهايى دارد كه نتايج آن بايد در سايه

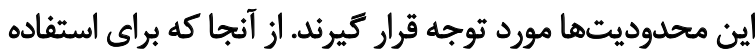

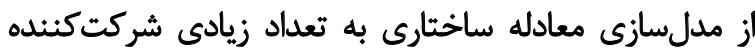

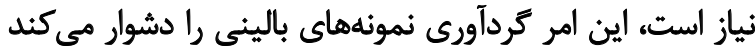

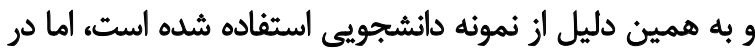
تعميم نتايج به نمونه بالينى بايد محتاط بود و تكرار مطالعه در الئر 


\section{References}

[1] Woody SR, Teachman BA. Intersection of disgust and fear: Normative and pathological views. Clinical Psychology: Science and Practice. 2000; 7(3):291-311. [DOI:10.1093/clipsy.7.3.291]

[2] Olatunji BO, Ebesutani C, Kim J, Riemann BC, Jacobi DM. Disgust proneness predicts obsessive-compulsive disorder symptom severity in a clinical sample of youth: Distinctions from negative affect. Journal of Affective Disorders. 2017; 213:118-25. [DOI:10.1016/j.jad.2017.02.017] [PMID]

[3] Olatunji BO, Moretz MW, Wolitzky-Taylor KB, McKay D, McGrath PB, Ciesielski BG. Disgust vulnerability and symptoms of contamination-based OCD: Descriptive tests of incremental specificity. Behavior Therapy. 2010; 41(4):475-90. [DOI:10.1016/j. beth.2009.11.005] [PMID]

[4] Goetz AR, Lee HJ, Cougle JR, Turkel JE. Disgust propensity and sensitivity: Differential relationships with obsessive-compulsive symptoms and behavioral approach task performance. Journal of Obsessive-Compulsive and Related Disorders. 2013; 2(4):412-9. [DOI:10.1016/j.jocrd.2013.07.006]

[5] Moretz MW, McKay D. Disgust sensitivity as a predictor of obsessive-compulsive contamination symptoms and associated cognitions. Journal of Anxiety Disorders. 2008; 22(4):707-15. [DOI:10.1016/j.janxdis.2007.07.004] [PMID]

[6] Schienle A, Stark R, Walter B, Vaitl D. The connection between disgust sensitivity and blood-related fears, faintness symptoms, and obsessive-compulsiveness in a non-clinical sample. Anxiety, Stress \& Coping. 2003; 16(2):185-93. [DOI:10.1080/10615806.2003 .10382972]

[7] Olatunji BO, Berg H, Cox RC, Billingsley A. The effects of cognitive reappraisal on conditioned disgust in contamination-based OCD: An analogue study. Journal of Anxiety Disorders. 2017; 51:86-93. [DOI:10.1016/j.janxdis.2017.06.005] [PMID]

[8] Rozin P, Haidt J, McCauley CR. Disgust. In: Lewis M, HavilandJones JM, Barret LF, editors. Handbook of Emotions. $3^{\text {rd }}$ ed. New York: Guilford Press; 2008. p. 757-776. https://books.google. com/books?id=DFK1QwlrOUAC\&dq

[9] Haidt J, McCauley C, Rozin P. Individual differences in sensitivity to disgust: A scale sampling seven domains of disgust elicitors. Personality and Individual Differences. 1994; 16(5):701-13. [DOI:10.1016/0191-8869(94)90212-7]

[10] Rozin P, Fallon AE. A perspective on disgust. Psychological Review. 1987; 94(1):23-41. [DOI:10.1037/0033-295X.94.1.23] [PMID]

[11] Cisler JM, Adams TG, Brady RE, Bridges AJ, Lohr JM, Olatunji $\mathrm{BO}$. Unique affective and cognitive processes in contamination appraisals: Implications for contamination fear. Journal of Anxiety Disorders. 2011; 25(1):28-35. [DOI:10.1016/j.janxdis.2010.07.002] [PMID] [PMCID]

[12] Rozin P, Millman L, Nemeroff C. Operation of the laws of sympathetic magic in disgust and other domains. Journal of Personality and Social Psychology. 1986; 50(4):703-12. [DOI:10.1037/00223514.50.4.703]

[13] Rozin P, Markwith M, Nemeroff C. Magical contagion beliefs and fear of AIDS. Journal of Applied Social Psychology. 1992; 22(14):1081-92. [DOI:10.1111/j.1559-1816.1992.tb00943.x]

[14] Rozin P, Nemeroff C, Wane M, Sherrod A. Operation of the sympathetic magical law of contagion in interpersonal attitudes among Americans. Bulletin of the Psychonomic Society. 1989; 27(4):367-70. [DOI:10.3758/BF03334630]

[15] Tolin DF, Worhunsky P, Maltby N. Sympathetic magic in contamination-related OCD. Journal of Behavior Therapy and Experimental Psychiatry. 2004; 35(2):193-205. [DOI:10.1016/j. jbtep.2004.04.009] [PMID]

[16] Adams Jr TG, Cisler JM, Brady RE, Lohr JM. The effects of cognitive and affective priming on law of contagion appraisals. Journal of Experimental Psychopathology. 2012; 3(3):470-8. [DOI:10.5127/jep.025911]

[17] Wood SR, Tolin DF. The relationship between disgust sensitivity and avoidant behavior: Studies of clinical and nonclinical samples. Journal of Anxiety Disorders. 2002; 16(5):543-59. [DOI:10.1016/S0887-6185(02)00173-1]

[18] Jones MK, Menzies RG. The cognitive mediation of obsessivecompulsive handwashing. Behaviour Research and Therapy. 1997; 35(9):843-50. [DOI:10.1016/S0005-7967(97)00035-1]

[19] Mitte K. Trait-disgust vs. fear of contamination and the judgmental bias of contamination concerns. Journal of Behavior Therapy and Experimental Psychiatry. 2008; 39(4):577-86. [DOI:10.1016/j.jbtep.2008.01.005] [PMID]

[20] Verwoerd J, de Jong PJ, Wessel I, van Hout WJPJ. “If I feel disgusted, I must be getting ill": Emotional reasoning in the context of contamination fear. Behaviour Research and Therapy. 2013; 51(3):122-7. [DOI:10.1016/j.brat.2012.11.005] [PMID]

[21] Olatunji BO, Williams NL, Tolin DF, Abramowitz JS, Sawchuk CN, Lohr JM, et al. The Disgust Scale: Item analysis, factor structure, and suggestions for refinement. Psychological Assessment. 2007; 19(3):281-97. [DOI:10.1037/1040-3590.19.3.281] [PMID]

[22] Shams G, Foroughi E, Moretz MW, Olatunji BO. Factor structure and psychometric properties of the Persian disgust scale-revised: Examination of specificity to symptoms of obsessive-compulsive disorder. Psychology. 2013; 4(6):526-34. [DOI:10.4236/ psych.2013.46075]

[23] Fedotova NO. Psychological contagion: Alternative accounts, properties, and assessment [PhD. dissertation]. Philadelphia, PA: University of Pennsylvania; 2013. https:// repository.upenn.edu/ dissertations/AAI3565052/

[24] Fathi-Ashtiani A, Mohammadi B, Fathi-Ashtiani M. [Psychological tests (Persian)]. Tehran: Besat; 2016. http://opac.nlai.ir/ opac-prod/bibliographic/4519290

[25] Thordarson DS, Radomsky AS, Rachman S, Shafran R, Sawchuk CN, Ralph Hakstian A. The Vancouver Obsessional Compulsive Inventory (VOCI). Behaviour Research and Therapy. 2004; 42(11):1289-314. [DOI:10.1016/j.brat.2003.08.007] [PMID]

[26] Hodgson RJ, Rachman S. Obsessional-compulsive complaints. Behaviour Research and Therapy. 1977; 15(5):389-95. [DOI:10.1016/0005-7967(77)90042-0]

[27] Ghassemzadeh H, Shams G, Meysami AP, Karamghadiri N. Psychometric properties of the Persian version of the Vancouver Obsessional-Compulsive Inventory Inventory (VOCI) in Iranian non-clinical sample. Psychology. 2017; 8(1):206-25. [DOI:10.4236/ psych.2017.81012]

[28] Olatuni BO. Changes in disgust correspond with changes in symptoms of contamination-based OCD: A prospective examination of specificity. Journal of Anxiety Disorders. 2010; 24(3):313-7. [DOI:10.1016/j.janxdis.2010.01.003] [PMID] 
[29] Hu LT, Bentler PM. Cutoff criteria for fit indexes in covariance structure analysis: Conventional criteria versus new alternatives. Structural Equation Modeling: A Multidisciplinary Journal. 1999; 6(1):1-55. [DOI:10.1080/10705519909540118]

[30] Byrne BM. Structural equation modeling with EQS: Basic concepts, applications, and programming. $2^{\text {nd }}$ ed. Mahwah, NJ: Lawrence Erlbaum Associates; 2006. https:/ / books.google.com/ books?id=MXb8dDwidPgC\&dq

[31] Diamantopoulos A, Siguaw JA. Introducing LISREL: A Guide for the uninitiated. New York: Sage Publications; 2000. https:/ / books.google.com/books?id=sOyGAwAAQBAJ\&dq

[32] Adams TG, Cisler JM, Brady RE, Lohr JM, Olatunji BO. Preliminary psychometric evidence for distinct affective and cognitive mechanisms mediating contamination aversion. Journal of Psychopathology and Behavioral Assessment. 2013; 35(3):375-88. [DOI:10.1007/s10862-013-9343-8]

[33] Zanjani Z, Yaghubi H, Fata L, Shaiiri MR, Gholami M. [The mediating role of fear of contagion in explaining the relationship between disgust propensity and fear of contamination (Persian)]. Iranian Journal of Psychiatry and Clinical Psychology. 2018; 23(4):454-65. [DOI:10.29252/nirp.ijpcp.23.4.454]

[34] Cisler JM, Brady RE, Olatunji BO, Lohr JM. Disgust and obsessive beliefs in contamination-related OCD. Cognitive Therapy and Research. 2010; 34(5):439-48. [DOI:10.1007/s10608-0099253-y] [PMID] [PMCID]

[35] Matchett G, Davey GCL. A test of a disease-avoidance model of animal phobias. Behaviour Research and Therapy. 1991; 29(1):914. [DOI:10.1016/S0005-7967(09)80011-9]

[36] Rachman S. Pollution of the mind. Behaviour Research and Therapy. 1994; 32(3):311-4. [DOI:10.1016/0005-7967(94)90127-9]

[37] Shafran R, Thordarson DS, Rachman S. Thought-action fusion in obsessive compulsive disorder. Journal of Anxiety Disorders. 1996; 10(5):379-91. [DOI:10.1016/0887-6185(96)00018-7]

[38] Einstein DA, Menzies RG. The presence of magical thinking in obsessive compulsive disorder. Behaviour Research and Therapy. 2004; 42(5):539-49. [DOI:10.1016/S0005-7967(03)00160-8]

[39] Reuven O, Liberman N, Dar R. The Effect of physical cleaning on threatened morality in individuals with obsessive-compulsive disorder. Clinical Psychological Science. 2014; 2(2):224-9. [DOI:10.1177/2167702613485565]

[40] Connolly KM, Lohr JM, Olatunji BO, Hahn KS, Williams NL. Information processing in contamination fear: A covariation bias examination of fear and disgust. Journal of Anxiety Disorders. 2009; 23(1):60-8. [DOI:10.1016/j.janxdis.2008.03.017] [PMID]

[41] Nemeroff C, Rozin P. Sympathetic magical beliefs and kosher dietary practice: The interaction of rules and feelings. Ethos. 1992; 20(1):96-115. [DOI:10.1525/eth.1992.20.1.02a00040]

[42] Olatunji BO, Berg HE, Zhao Z. Emotion regulation of fear and disgust: Differential effects of reappraisal and suppression. Cognition and Emotion. 2017; 31(2):403-10. [DOI:10.1080/02699931.20 15.1110117] [PMID]

[43] Fink J, Pflugradt E, Stierle C, Exner C. Changing disgust through imagery rescripting and cognitive reappraisal in contamination-based obsessive-compulsive disorder. Journal of Anxiety Disorders. 2018; 54;36-48. [DOI:10.1016/j.janxdis.2018.01.002] 
This Page Intentionally Left Blank 